\title{
Regularity of Local Times Associated with Volterra-Lévy Processes and Path-Wise Regularization of Stochastic Differential Equations
}

\author{
Fabian A. Harang ${ }^{1}$. Chengcheng Ling ${ }^{2}$ \\ Received: 6 July 2020 / Revised: 11 June 2021 / Accepted: 18 June 2021 / \\ Published online: 16 July 2021 \\ (c) The Author(s) 2021
}

\begin{abstract}
We investigate the space-time regularity of the local time associated with VolterraLévy processes, including Volterra processes driven by $\alpha$-stable processes for $\alpha \in$ $(0,2]$. We show that the spatial regularity of the local time for Volterra-Lévy process is $\mathbb{P}$-a.s. inverse proportional to the singularity of the associated Volterra kernel. We apply our results to the investigation of path-wise regularizing effects obtained by perturbation of ordinary differential equations by a Volterra-Lévy process which has sufficiently regular local time. Following along the lines of Harang and Perkowski (2020), we show existence, uniqueness and differentiability of the flow associated with such equations.
\end{abstract}

Keywords Stochastic differential equations · Lévy process · Volterra process . Regularization by noise - Occupation measure $\cdot$ Local time $\cdot$ Young integral . Stochastic Sewing Lemma

Mathematics Subject Classification (2020) Primary 60H10 · 35R09; Secondary 60G51

\section{Contents}

1 Introduction . . . . . . . . . . . . . . . . . . . . . . . . . . 1707

1.1 Main Results . . . . . . . . . . . . . . . . . . . . . . . . . . . . . . . . 1709

1.2 Structure of the Paper . . . . . . . . . . . . . . . . . . . . . 1711

$凶$ Chengcheng Ling

cling@math.uni-bielefeld.de

Fabian A. Harang

fabianah@math.uio.no

1 Department of Mathematics, University of Oslo, P.O. Box 1053, Blindern 0316, Oslo, Norway

2 Faculty of Mathematics, Bielefeld University, 33615 Bielefeld, Germany 
1.3 Notation . . . . . . . . . . . . . . . . . . . . . . . . . . . . 1711

2 Occupation Measures and Local Times, and Distributions . . . . . . . . . . . . . . . 1712

2.1 Occupation Measure and Local Times . . . . . . . . . . . . . . . . . . . . . . 1712

2.2 Besov Spaces and Distributions . . . . . . . . . . . . . . . . . . . . . . . . . . . . . . 1714

3 Volterra-Lévy Process . . . . . . . . . . . . . . . . . . . . . . . . . . . . . 1714

4 Regularity of the Local Time Associated with Volterra-Lévy Processes . . . . . . . . . . . . 1719

4.1 Local Non-determinism Condition for Volterra-Lévy Process . . . . . . . . . . . . . . . . 1719

4.2 Regularity of the Local Time . . . . . . . . . . . . . . . . . . . . . . . . . 1721

5 Regularization of ODEs Perturbed by Volterra-Lévy Processes . . . . . . . . . . . . . . 1727

Appendix A. Stochastic Sewing Lemma . . . . . . . . . . . . . . . . . . . . . . . . . . 1730

Appendix B. Nonlinear Young integration and equations _ . . . . . . . . . . . . . . 1731

References . . . . . . . . . . . . . . . . . . . . . . . . . . . . 1734

\section{Introduction}

Occupation measures and local times associated with $d$-dimensional paths $\left(p_{t}\right)_{t \in[0, T]}$ have received much attention over the past decades from both in the analytical and the probabilistic community. The occupation measure essentially quantifies the amount of time the path $p$ spends in a given set, i.e. for a Borel set $A \in \mathcal{B}\left(\mathbb{R}^{d}\right)$ the occupation measure is given by

$$
\mu_{t}(A)=\lambda\left\{s \in[0, t] \mid p_{s} \in A\right\}
$$

where $\lambda$ is the Lebesgue measure on $\mathbb{R}$. The local time is given as the Radon-Nikodym derivative of the occupation measure with respect to the Lebesgue measure. The existence of the local time is generally not assured without some further knowledge of the path $p$, and the existence of the local time associated with the Weierstrass function, and other deterministic fractal like paths, is, to the best of our knowledge, still considered an open question. However, when $\left(p_{t}\right)_{t \in[0, T]}$ is a stochastic process, existence of the local time can often be proved using probabilistic techniques, and much research has been devoted to this aim, see, e.g. [14] and the references therein for a comprehensive overview. Knowledge of probabilistic and analytic properties of the local time becomes useful in a variety problems arising in analysis. For example, given a measurable path $p$ with an existing local time, the following formula holds

$$
\int_{0}^{t} b\left(x-p_{s}\right) \mathrm{d} s=b * L_{t}(x)
$$

where $*$ denotes convolution, and $L:[0, T] \times \mathbb{R}^{d} \rightarrow \mathbb{R}_{+}$is the local time associated with $p$. Thus, analytical or probabilistic questions relating to the left hand side integral can often be answered with the knowledge of the probabilistic and analytic properties of the local time $L$.

In this article, we will study regularity properties of the local time associated with Volterra-Lévy processes given on the form 


$$
z_{t}=\int_{0}^{t} k(t, s) \mathrm{d} \mathscr{L}_{s}, \quad t \in[0, T],
$$

where $k(t, \cdot) \in L^{\alpha}([0, t])$ for all $t \in[0, T]$ with $\alpha \in(0,2]$, and $\mathscr{L}$ is a Lévy process on a filtered probability space $(\Omega, \mathcal{F}, \mathbb{P})$. In the case, when $\mathscr{L}=B$ is a Brownian motion, then joint regularity in time and space of the local time associated with Volterra processes has received some attention in recent years as this knowledge can be applied towards regularization of ordinary differential equations (ODEs) by noise $[6,12,13,15]$, as discussed in detail below. Furthermore, in [13], the authors investigated the regularity of the local time associated with $\alpha$-stable processes, i.e. when the kernel $k \equiv 1$, and $\mathscr{L}$ is an $\alpha$-stable process. One goal of this article is therefore to extend these results to the general case of Volterra-Lévy processes, as well as apply this to the regularization by noise procedure. Towards this end, we formulate a simple local non-determinism condition for these processes, which will be used to determine the regularity of the local time. The regularity of the local time is then proved in Sobolev space, by application of the recently developed stochastic sewing lemma [19], similarly as done for Gaussian Volterra processes in [15]. By embedding, it follows that the local time is also contained in a wide range of Besov spaces.

As an application of our results on regularity of the local time, we show existence and pathwise uniqueness of stochastic differential equations (SDEs) of the form

$$
\frac{\mathrm{d}}{\mathrm{d} t} x_{t}=b\left(x_{t}\right)+\frac{\mathrm{d}}{\mathrm{d} t} z_{t}, \quad x_{0}=\xi \in \mathbb{R}^{d}
$$

even when $b$ is a Besov-distribution (the exact regularity requirement of $z$ and $b$ will be given in Sect. (1.1) below). It is well known that certain stochastic processes provide a regularizing effect on SDEs on the form of (1.2). By this, we mean that if the process $\left(z_{t}\right)_{t \in[0, T]}$ is given on some explicit form, (1.2) might be well posed, even when $b$ does not satisfy the usual assumption of Lipschitz and linear growth. In fact, in [6], the authors show that if $z$ is given as a sample path of a fractional Brownian motion with Hurst index $H \in(0,1)$, Eq. (1.2) is well posed and has a unique solution even when $b$ is only a distribution in the generalized Besov-Hölder space $\mathcal{C}^{\beta}$ with $\beta<\frac{1}{2 H}-2$. More recently, Perkowski and one of the authors of the current article in [15] proved that there exists a certain class of continuous Gaussian processes with exceptional regularization properties. In particular, if $z$ in (1.2) is given as a path of such a process, then a unique solution exists to (1.2) (where the equation is understood in the pathwise sense), for any $b \in \mathcal{C}^{\beta}$ with $\beta \in \mathbb{R}$. Moreover, the flow map $\xi \mapsto x_{t}(\xi)$ is infinitely differentiable. We then say that the path $z$ is infinitely regularizing. Not long after this result was published, Galeati and Gubinelli [12], showed that in fact almost all continuous paths are infinitely regularizing by using the concept of prevalence. Furthermore, the regularity assumption on $b$ was proven to be inverse proportional to the irregularity of the continuous process $z$. In fact, this statement holds in a purely deterministic sense, see, e.g. [12, Thm. 1]. The main ingredient in this approach to regularization by noise is to formulate the ODE/SDE into a nonlinear Young equation, 
involving a nonlinear Young integral, as was first described in [6]. This reformulation allows one to construct integrals, even in the case when traditional integrals (Riemann, Lebesgue, etc.) do not make sense. A particular advantage of this theory is furthermore that the framework itself does not rely on any probabilistic properties of the processes, such as Markov or martingale properties. This makes this framework particularly suitable when considering SDEs where the additive stochastic process is of a more exotic type. As is demonstrated in the current paper, the framework is well suited to study SDEs driven by Volterra-Lévy processes, which is a class of processes difficult to analyse using traditional probabilistic techniques. We believe that this powerful framework can furthermore be applied towards analysing several interesting problems relating to ill-posed SDEs and ODEs in the future.

Historically, the investigation of similar regularising effects for SDEs with general Lévy noise seems to have received less attention compared to the case when the SDE (1.2) is driven by a continuous Gaussian process. Of course, the general structure of the Lévy noise excludes several techniques which has previously been applied in the Gaussian case. However, much progress has been made also on this front when the equation has jump type noise, and although several interesting results deserve to be mentioned, we will only discuss here some the most recent results and refer the reader to $[4,9,10,18,30]$ for further results. In [22], Priola showed that (1.2) has a path-wise unique strong solution (in a probabilistic sense) when $z=\mathscr{L}$ is a symmetric $\alpha$-stable process with $\alpha \in(0,2)$ and $b$ is a bounded $\beta$-Hölder continuous function of order $\beta>1-\frac{\alpha}{2}$. In [23], this result was put in the context of path-by-path uniqueness suggested by Davie [7]. More recently, in [8] the authors prove that the martingale problem associated with (1.2) is well posed, even when $b$ is only assumed to be bounded and continuous, in the case when $z=\mathscr{L}$ is an $\alpha$-stable process with $\alpha=1$ (being the critical case). Further in [2], the authors show strong existence and uniqueness of (1.2) when $z=\mathscr{L}$ is an one-dimensional $\alpha$-stable process, and $b \in \mathcal{C}^{\beta}$ with $\beta>\frac{1}{2}-\frac{\alpha}{2}$. Thus, allowing here for possibly distributional coefficients $b$ when $\alpha$ is sufficiently large (i.e. greater than 1). Our results can be seen as an extension of the last result to a purely pathwise setting, and to the case of general Volterra-Lévy processes. Similarly as seen in the Gaussian case, the choice of Volterra kernel then dictates the regularity $\beta \in \mathbb{R}$ of the distribution $b \in \mathcal{C}^{\beta}$ that can be considered to still obtain existence and uniqueness.

\subsection{Main Results}

We present here the main results to be proven in this article. The first result provides a simple condition to show regularity of the local time associated with Volterra-Lévy processes.

Theorem 1 Let $\left(\mathscr{L}_{t}\right)_{t \in[0, T]}$ be a Lévy process on a filtered probability space $(\Omega, \mathcal{F}, \mathbb{P})$, with characteristic $\psi: \mathbb{R}^{d} \rightarrow \mathbb{C}$, and let $k$ be a real valued and possibly singular Volterra kernel satisfying for $t \in[0, T], k(t, \cdot) \in L^{\alpha}([0, t])$ with $\alpha \in(0,2]$. Define the Volterra-Lévy process $\left(z_{t}\right)_{t \in[0, T]}$ by $z_{t}:=\int_{0}^{t} k(t, s) \mathrm{d} \mathscr{L}_{s}$, where the integral is defined in Definition 17. Suppose that the characteristic triplet and the Volterra kernel 
satisfy for some $\zeta>0$ and $\alpha \in(0,2]$

$$
\inf _{t \in[0, T]} \inf _{s \in[0, t]} \inf _{\xi \in \mathbb{R}^{d}} \frac{\int_{s}^{t} \psi(k(t, r) \xi) \mathrm{d} r}{(t-s)^{\zeta}|\xi|^{\alpha}}>0 .
$$

If $\zeta \in\left(0, \frac{\alpha}{d}\right)$, then there exists a $\gamma>\frac{1}{2}$ such that the local time $L: \Omega \times[0, T] \times \mathbb{R}^{d} \rightarrow$ $\mathbb{R}_{+}$associated with $z$ is contained in $\mathcal{C}^{\gamma}\left([0, T] ; H^{\kappa}\left(\mathbb{R}^{d}\right)\right)$ for any $\kappa<\frac{\alpha}{2 \zeta}-\frac{d}{2}, \mathbb{P}$-a.s..

Corollary 2 There exists a class of Volterra-Lévy processes $z_{t}=\int_{0}^{t} k(t, s) \mathrm{d} \mathscr{L}_{s}$ such that for each $t \in[0, T]$, its associated local time $L_{t}$ is a test function. More precisely, we have that $(t, x) \mapsto L_{t}(x) \in \mathcal{C}^{\gamma}\left([0, T] ; \mathcal{D}\left(\mathbb{R}^{d}\right)\right) \mathbb{P}$-a.s. for any $\gamma \in(0,1)$. Here, $\mathcal{D}\left(\mathbb{R}^{d}\right)$ denotes the space of test functions on $\mathbb{R}^{d}$.

See Example 32, (iv) for proof of this corollary.

Inspired by $[6,13,15]$, we apply the result on regularity of the local time to prove regularization of SDEs by Volterra-Lévy noise. Since we will allow the coefficient $b$ in (1.2) to be distributional-valued, it is not a priori clear what we mean by a solution. Indeed, since the integral $\int_{0}^{t} b\left(x_{s}\right) \mathrm{d} s$ is not well defined in a Riemann or Lebesgue sense if $b$ is truly distributional, it is not a priori clear how to make sense of (1.2). We therefore begin with the following definition of a solution, which is in line with the definition of pathwise solutions to SDEs used in $[6,13,15]$.

Definition 3 Consider a Volterra-Lévy process $z$ given as in (1.1) with measurable paths, and associated local time $L$. Let $b \in \mathcal{S}^{\prime}\left(\mathbb{R}^{d}\right)$ be a distribution such that $b * L \in$ $\mathcal{C}^{\gamma}\left([0, T] ; \mathcal{C}^{2}\left(\mathbb{R}^{d}\right)\right)$ for some $\gamma>\frac{1}{2}$. Then, for any $\xi \in \mathbb{R}^{d}$ we say that $x$ is a solution to

$$
x_{t}=\xi+\int_{0}^{t} b\left(x_{s}\right) \mathrm{d} s+z_{t}, \quad \forall t \in[0, T],
$$

if and only if $x-z \in \mathcal{C}^{\gamma}\left([0, T] ; \mathbb{R}^{d}\right)$, and there exists a $\theta \in \mathcal{C}^{\gamma}\left([0, T] ; \mathbb{R}^{d}\right)$ such that $\theta=x-z$, and $\theta$ solves the nonlinear Young equation

$$
\theta_{t}=\xi+\int_{0}^{t} b * \bar{L}_{\mathrm{d} r}\left(\theta_{r}\right), \quad \forall t \in[0, T]
$$

Here, $\bar{L}_{t}(z)=L_{t}(-z)$ where $L$ is the local time associated with $\left(z_{t}\right)_{t \in[0, T]}$, and the integral is interpreted in the nonlinear Young sense, described in Lemma 38.

Theorem 4 Suppose $\left(z_{t}\right)_{t \in[0, T]}$ is a Volterra-Lévy process such that its associated local time $L \in \mathcal{C}^{\gamma}\left([0, T] ; H^{\kappa}\right)$ for some $\kappa>0$ and $\gamma>\frac{1}{2}$, $\mathbb{P}$-a.s.. Then, for any 
$b \in H^{\beta}\left(\mathbb{R}^{d}\right)$ with $\beta>2-\kappa$, there exists a unique pathwise solution to the equation

$$
x_{t}=\xi+\int_{0}^{t} b\left(x_{s}\right) \mathrm{d} s+z_{t}, \quad \forall t \in[0, T],
$$

where the solution is interpreted in sense of Definition 3. Moreover, if $\beta>n+1-\kappa$ for some $n \in \mathbb{N}$, then the flow mapping $\xi \mapsto x_{t}(\xi)$ is $n$-times continuously differentiable.

\subsection{Structure of the Paper}

In Sect. 2, we recall some basic aspects from the theory of occupation measures, local times, and Sobolev/Besov distribution spaces. Section 3 introduces a class of Volterra processes where the driving noise is given as a Lévy process. We show a construction of such processes, even in the case of singular Volterra kernels, and discuss conditions under which the process is continuous in probability. Several examples of Volterra-Lévy processes are given, including a rough fractional $\alpha$-stable process, with $\alpha \in[1,2)$. In Sect. 4, we provide some sufficient conditions for the characteristics of Volterra-Lévy processes such that their associated local time exists and is $\mathbb{P}$-a.s. contained in a Hölder-Sobolev space of positive regularity. At last, we apply the concept of local times in order to prove regularization by noise for SDEs with additive Volterra-Lévy processes. Here, we apply the framework of nonlinear Young equations and integration, and thus, our results can truly be seen as pathwise, in the "rough path" sense. An appendix is included in the end, where statements and proofs of some auxiliary results are given.

\subsection{Notation}

For a fixed $T>0$, we will denote by $x_{t}$ the evaluation of a function at time $t \in[0, T]$, and write $x_{s, t}=x_{t}-x_{s}$. For some $n \in \mathbb{N}$, we define

$$
\Delta_{T}^{n}:=\left\{\left(s_{1}, \ldots, s_{n}\right) \in[0, T]^{n} \mid s_{1} \leq \cdots \leq s_{n}\right\}
$$

To avoid confusion, the letter $\mathscr{L}$ will be used to denote a Lévy process, while $L$ will be used to denote the local time of a process. For $\gamma \in(0,1)$ and a Banach space $E$, the space $\mathcal{C}_{T}^{\gamma} E:=\mathcal{C}^{\gamma}([0, T] ; E)$ is defined to be the space of functions $f:[0, T] \rightarrow E$ which is Hölder continuous of order $\gamma$. The space is equipped with the standard seminorm

$$
\|f\|_{\gamma}:=\sup _{s \neq t \in[0, T]} \frac{\left\|f_{t}-f_{s}\right\|_{E}}{|t-s|^{\gamma}}
$$

and note that under the mapping $f \mapsto\left|f_{0}\right|+\|f\|_{\gamma}$ the space $\mathcal{C}_{T}^{\gamma} E$ is a Banach space.

We let $\mathcal{S}\left(\mathbb{R}^{d}\right)$ denote the Schwartz space of rapidly decreasing functions on $\mathbb{R}^{d}$, and $\mathcal{S}^{\prime}\left(\mathbb{R}^{d}\right)$ its dual space. Given $f \in \mathcal{S}\left(\mathbb{R}^{d}\right)$, let $\mathscr{F} f$ be the Fourier transform of $f$ 
defined by

$$
\mathscr{F} f(\xi):=(2 \pi)^{-d / 2} \int_{\mathbb{R}^{d}} \mathrm{e}^{-i\langle\xi, x\rangle} f(x) \mathrm{d} x .
$$

Let $s$ be a real number. The Sobolev space $H^{s}\left(\mathbb{R}^{d}\right)$ consists of distributions $f \in \mathcal{S}^{\prime}\left(\mathbb{R}^{d}\right)$ such that $\mathscr{F} f \in L_{\text {loc }}^{2}\left(\mathbb{R}^{d}\right)$ and

$$
\|f\|_{H^{s}}^{2}:=\int_{\mathbb{R}^{d}}\left(1+|\xi|^{2}\right)^{s}|\mathscr{F} f(\xi)|^{2} \mathrm{~d} \xi<\infty
$$

For $\alpha>0$, if $\int_{0}^{T}|f(s)|^{\alpha} \mathrm{d} s<\infty$, then we say $f \in L^{\alpha}([0, T])$.

\section{Occupation Measures and Local Times, and Distributions}

This section is devoted to give some background on the theory of occupation measures and local times, as well as definitions of Sobolev and Besov spaces, which will play a central role throughout this article.

\subsection{Occupation Measure and Local Times}

The occupation measure associated with a process $\left(x_{t}\right)_{t \in[0, T]}$ gives information about the amount of time the process spends in a given set. Formally, we define the occupation measure $\mu$ associated with $\left(x_{t}\right)_{t \in[0, T]}$ evaluated at $t \in[0, T]$ by

$$
\mu_{t}(A)=\lambda\left\{s \leq t \mid x_{s} \in A\right\}
$$

where $\lambda$ denotes the Lebesgue measure. The Local time $L$ associated with $x$ is then the Radon-Nikodym derivative with of $\mu$ with respect to the Lebesgue measure(as long as this exists). We therefore give the following definition.

Definition 5 Consider a process $x:[0, T] \rightarrow \mathbb{R}^{d}$ be a process, and let $\mu$ denote the occupation measure of $x$. If there exists a function $L:[0, T] \times \mathbb{R}^{d} \rightarrow \mathbb{R}_{+}$such that

$$
\mu_{t}(A)=\int_{A} L_{t}(z) \mathrm{d} z, \quad \text { for } \quad A \in \mathcal{B}\left(\mathbb{R}^{d}\right),
$$

then we say that $L$ is the local time associated with the process $\left(x_{t}\right)_{t \in[0, T]}$.

Remark 6 The interpretation of the local time $L_{t}(z)$ is the time spent by the process $x:[0, T] \rightarrow \mathbb{R}^{d}$ at a given point $z \in \mathbb{R}^{d}$. Thus, the study of this object has received much attention from people investigating both probabilistic and path-wise properties of stochastic processes. For purely deterministic processes $\left(x_{t}\right)_{t \in[0, T]}$, the local time 
might still exist; however, as discussed in [15], if $x$ is a Lipschitz path, there exists at least two discontinuities of the mapping $z \mapsto L_{t}(z)$. On the other hand, it is well known (see [14]) that the local time associated with the trajectory of a one-dimensional Brownian motion is $\frac{1}{2}$-Hölder regular in its spatial variable (a.s.). More generally, for the trajectory of a fractional Brownian motion with Hurst index $H \in(0,1)$, we know that its local time $L$ is contained in $H^{\kappa}$ (a.s.) for $\kappa<\frac{1}{2 H}-\frac{d}{2}$, while still preserving Hölder regularity in time. This clearly shows that the more irregular the trajectory of the fractional Brownian motion is, the more regularity we obtain in the local time associated with this trajectory. In this case, the regularity of the local time can therefore be seen as an irregularity condition. This heuristic has recently been formalized in [13]. There, the authors show that if the local time associated with a continuous path $\left(x_{t}\right)_{t \in[0, T]}$ is regular (i.e. Hölder continuous or better) in space, then $x$ is truly rough, in the sense of [11]. More recently, the authors of [15] showed that the local time associated with trajectories of certain particularly irregular Gaussian processes (for example the log-Brownian motion) is infinitely differentiable in space, and almost Lipschitz in time. In the current article, we will extend this analysis to Lévy processes.

The next proposition will be particularly interesting towards applications in differential equations, and which we will use in subsequent sections.

Proposition 7 (Local time formula) Let $b$ be a measurable function, and suppose $\left(x_{t}\right)_{t \in[0, T]}$ is a process with associated local time L. Then, the following formula holds for any $\xi \in \mathbb{R}^{d}$ and $(s, t) \in \Delta_{T}^{2}$

$$
\int_{S}^{t} b\left(\xi+x_{r}\right) \mathrm{d} r=b * \bar{L}_{s, t}(\xi),
$$

where $\bar{L}_{t}(z)=L_{t}(-z)$ and $L_{s, t}=L_{t}-L_{s}$ denotes the increment.

A proof of this statement follows directly from the definition of the local time, see [14, Thm. 6.4] for further details.

Remark 8 It is readily seen that, formally, the local time can be expressed in the following way for $\xi \in \mathbb{R}^{d}$ and $(s, t) \in \Delta_{T}^{2}$

$$
L_{s, t}(\xi)=\int_{s}^{t} \delta\left(\xi-X_{r}\right) \mathrm{d} r,
$$

where $\delta$ is the Dirac distribution.

Remark 9 For future reference, we also recall here that the Dirac distribution $\delta$ is contained in the in-homogeneous Sobolev space $H^{-\frac{d}{2}-\epsilon}$ for any $\epsilon>0$ (See, e.g. [3, Remark 1.54]). 


\subsection{Besov Spaces and Distributions}

Before introducing the notion of Besov spaces, we give a definition of the PaleyLittlewood blocks, which plays a central role in the construction of these spaces.

Definition 10 (Paley-Littlewood blocks) For $j \in \mathbb{N}, \rho_{j}:=\rho\left(2^{-j}\right.$.) where $\rho$ is a smooth function supported on an annulus $\mathcal{A}:=\left\{x \in \mathbb{R}^{d}: \frac{4}{3} \leq|x| \leq \frac{8}{3}\right\}$ and $\rho_{-1}$ is a smooth function supported on the ball $B_{\frac{4}{3}}$. Then, $\left\{\rho_{j}\right\}_{j \geq-1}$ is a partition of unity ([3]). For $j \geq-1$ and some $f \in \mathcal{S}^{\prime}$, we define the Paley-Littlewood blocks $\Delta_{j}$ in the following way

$$
\Delta_{j} f=\mathscr{F}^{-1}\left(\rho_{j} \mathscr{F} f\right) .
$$

Definition 11 For $\alpha \in \mathbb{R}$ and $p, q \in[1, \infty]$, the in-homogeneous Besov space $\mathcal{B}_{p, q}^{\alpha}$ is defined by

$$
\mathcal{B}_{p, q}^{\alpha}=\left\{f \in \mathcal{S}^{\prime} \mid\|f\|_{B_{p, q}^{\alpha}}:=\left(\sum_{j \geq-1} 2^{j q \alpha}\left\|\Delta_{j} f\right\|_{L^{p}\left(\mathbb{R}^{d}\right)}^{q}\right)^{\frac{1}{q}}<\infty\right\} .
$$

We will typically write $\mathcal{C}^{\alpha}:=\mathcal{B}_{\infty, \infty}^{\alpha}$. Besides, by the definition of the partition of unity and Fourier-Plancherel formula ([3, Examples p99]), the Besov space $\mathcal{B}_{2,2}^{\alpha}$ coincides with Sobolev space $H^{\alpha}$.

Remark 12 We will work with regularity of the local time in the Sobolev space $H^{\kappa}$. However, towards applications to regularization by noise in SDEs, we will also encounter Besov spaces, through Young's convolution inequality. We therefore give a definition of these spaces here. Of course, through Besov embedding, $H^{\kappa} \hookrightarrow B_{p, q}^{\kappa-\left(\frac{d}{2}-\frac{d}{p}\right)}$ for any $p, q \in[2, \infty]$ and $\kappa \in \mathbb{R}$, (e.g. [3, Prop. 2.20]), and thus, our results imply that the local time is also included in these Besov spaces. We will, however, not specifically work in this setting to avoid extra confusion, but refer the reader to $[12,13]$ for a good overview of regularity of the local time associated with Gaussian processes in such spaces.

\section{Volterra-Lévy Process}

In this section, we give a brief introduction on Lévy processes and stochastic integral for a Volterra kernel with respect to a Lévy process. General references for this part are [28, Chp. 4] and [1, Chp. 2, Chp. 4]. In Sect. 3.1, we give the definition of VolterraLévy processes (with possibly singular kernels) and obtain the associated characteristic function. Particularly, our framework includes Volterra processes driven by symmetric $\alpha$-stable noise. In the end, we provide several examples of Volterra-Lévy processes, including the fractional $\alpha$-stable process.

We begin to provide a definition of Lévy processes, as well as a short discussion on a few important properties. 
Definition 13 (Lévy process) Let $T>0$ be fixed. We say that a càdlàg and $\left(\mathcal{F}_{t}\right)$ adapted stochastic process $\left(\mathscr{L}_{t}\right)_{t \in[0, T]}$ defined on a complete probability space $\left(\Omega, \mathcal{F},\left(\mathcal{F}_{t}\right)_{t \in[0, T]}, \mathbb{P}\right)$, and which satisfies the usual assumptions is a Lévy process if the following properties hold:

(i) $\mathscr{L}_{0}=0$ (P्P-a.s.).

(ii) $\mathscr{L}$ has independent and stationary increments.

(iii) $\mathscr{L}$ is continuous in probability, i.e. for all $\epsilon>0$, and all $s>0$,

$$
\lim _{t \rightarrow s} \mathbb{P}\left(\left|\mathscr{L}_{t}-\mathscr{L}_{s}\right|>\epsilon\right)=0
$$

Furthermore, let $v$ be a $\sigma$-finite measure on $\mathbb{R}^{d}$. We say that it is a Lévy measure if

$$
v(\{0\})=0, \quad \int_{\mathbb{R}^{d}}\left(1 \wedge|x|^{2}\right) v(\mathrm{~d} x)<\infty .
$$

Remark 14 A known description of Lévy process is Lévy-Khintchine formula: for a $d$-dimensional Lévy process $\mathscr{L}$, the characteristic function $\psi$ of $\mathscr{L}$ verifies that for $t \geq 0$, there exists a vector $a \in \mathbb{R}^{d}$, a positive definite symmetric $d \times d$ matrix $\sigma$ and a Lévy measure $v$ such that the characteristic function is given by $\mathbb{E}\left[\mathrm{e}^{i\left\langle\xi, \mathscr{L}_{t}\right\rangle}\right]=\mathrm{e}^{-t \psi(\xi)}$ with

$$
\psi(\xi)=-i\langle a, \xi\rangle+\frac{1}{2}\langle\xi, \sigma \xi\rangle-\int_{\mathbb{R}^{d}-\{0\}}\left(\mathrm{e}^{i\langle\xi, x\rangle}-1-i\langle\xi, x\rangle 1_{|x| \leq 1}(x)\right) \nu(\mathrm{d} x) .
$$

Here, the triple $(a, \sigma, v)$ is called the characteristic of the random variable $\mathscr{L}_{1}$.

The typical examples for Lévy processes are the case when the Lévy triplet is given by $(0, \sigma, 0)$, resulting in a Brownian motion. Another typical example is when the characteristic triplet is given by $(0,0, v)$ and the Lévy measure $v$ defines an $\alpha$-stable process. We provide the following definition for this class of processes.

Definition 15 (Standard $\alpha$-stable process) If a $d$-dimensional Lévy process $\left(\mathscr{L}_{t}\right)_{t \geq 0}$ has the following characteristic function

$$
\psi(\xi)=c_{\alpha}|\xi|^{\alpha}, \quad \xi \in \mathbb{R}^{d}
$$

with $\alpha \in(0,2]$ and some positive constant $c_{\alpha}$, then we say $\left(\mathscr{L}_{t}\right)_{t \geq 0}$ is a standard $\alpha$-stable process.

We now move on to the construction of Volterra-Lévy processes, given of the form

$$
z_{t}=\int_{0}^{t} k(t, s) \mathrm{d} \mathscr{L}_{s}, \quad t \in[0, T]
$$


Of course in the case when $\left(\mathscr{L}_{t}\right)_{t \in[0, T]}$ is a Gaussian process, or even a square integrable martingale, the construction of such a stochastic integral is by now standard, and $z$ is constructed as an element in $L^{2}(\Omega)$ given that $k(t, \cdot) \in L^{2}([0, t])$ for all $t \in[0, T]$, see, e.g. [24]. However, in the case when $\mathscr{L}$ is not square integrable, then the construction of $z$ as a stochastic integral is not as straight forward. However, several articles discuss also this construction in the case of $\alpha$-stable processes, which would be sufficient for our purpose. The next remark gives only a brief overview on this construction, and we therefore ask the interested reader to consult the given references for further details on the construction.

Remark 16 Consider a symmetric $\alpha$-stable process $\mathscr{L}$ with $\alpha \in(0,2)$. From [28, Ex. 25.10, p162], we know that $\mathbb{E}\left[\left|\mathscr{L}_{t}\right|^{p}\right]=C t^{p / \alpha}$ for any $-1<p<\alpha$ and $t \in[0, T]$, and thus, the process is not square integrable and the standard "Itô type" construction of the Volterra process in 3.2 cannot be applied. However, in [27, Chp. 3.2-3.12] the authors propose several different ways of constructing integral $\int_{0}^{t} k(t, s) \mathrm{d} \mathscr{L}_{s}$ given that $k(t, \cdot) \in L^{\alpha}([0, t])$. In particular, in [27, Chp. 3.6] it is shown that the Volterrastable process below is well-defined and exists in $L^{p}(\Omega)$ for any $p<\alpha$, given that the kernel $k(t, \cdot) \in L^{\alpha}([0, t])$ for all $t \in[0, T]$. In fact, in the case when $\mathscr{L}$ is a symmetric $\alpha$-stable process, it is known that for any $0<p<\alpha$

$$
\left(\mathbb{E}\left[\left|\int_{0}^{t} k(t, s) \mathrm{d} \mathscr{L}_{s}\right|^{p}\right]\right)^{\frac{1}{p}} \simeq p, \alpha, d\left(\int_{0}^{t}|k(t, s)|^{\alpha} \mathrm{d} s\right)^{\frac{1}{\alpha}},
$$

where $\simeq_{p, \alpha, d}$ means that they differ up to a constant depending on $p, \alpha$ and $d$ (recall that $d$ is the dimension of $\mathscr{L}$ ). See, e.g. [26] and the references therein for more details on this relation and the construction of such integrals.

The above discussion yields the following definition of the Volterra-Lévy process.

Definition 17 (Volterra-Lévy process) Fix $T>0$, and let $\left(\mathscr{L}_{t}\right)_{t \in[0, T]}$ be a Lévy process as given in Definition 13. For a given kernel $k: \Delta_{T}^{2} \rightarrow \mathbb{R}$ with the property that for any $t \in[0, T], k(t, \cdot) \in L^{\beta}([0, t])$ with $\beta \in(0,2]$, define

$$
z_{t}=\int_{0}^{t} k(t, s) \mathrm{d} \mathscr{L}_{s}, \quad t \geq 0
$$

where the integral is constructed in $L^{p}(\Omega)$ sense for $p \leq \beta$, as discussed above. Then, we call the stochastic process $\left(z_{t}\right)_{t \in[0, T]}$ a Volterra-Lévy process, where $\mathscr{L}$ is the associated Lévy process to $z$ and $k$ is called the Volterra kernel.

Based on this definition, it is readily to have the following known property of Volterra-Lévy process, see, e.g. [25]. 
Proposition 18 Let $\left(\mathscr{L}_{t}\right)_{t \in[0, T]}$ be a Lévy process on a probability space $(\Omega, \mathcal{F}, \mathbb{P})$, such that $\mathbb{E}\left[\left|\mathscr{L}_{t}\right|^{p}\right]<\infty$ for all $0<p<\beta$ where $\beta \in(0,2]$. If $k(t, \cdot) \in L^{\beta}([0, t])$ for any $t \in[0, T]$, then the Volterra-Lévy process $\left(z_{t}\right)_{t \in[0, T]}$ given by

$$
z_{t}=\int_{0}^{t} k(t, s) \mathrm{d} \mathscr{L}_{s}
$$

is well defined as an element of $L^{p}(\Omega)$ for any $0<p<\beta$. For $0 \leq s \leq t \leq T$, the characteristic function of $z$ is given by

$$
\mathbb{E}\left[\exp \left(i\left\langle\xi, z_{t}\right\rangle\right)\right]=\exp \left(-\int_{0}^{t} \psi(k(t, s) \xi) \mathrm{d} s\right)
$$

and the conditional characteristic function is given by

$$
\mathbb{E}\left[\exp \left(i\left\langle\xi, z_{t}\right\rangle\right) \mid \mathcal{F}_{s}\right]=\mathcal{E}_{0, s, t}(\xi) \exp \left(-\int_{s}^{t} \psi(k(t, r) \xi) \mathrm{d} r\right)
$$

where $\mathcal{E}_{0, s, t}(\xi):=\exp \left(i\left\langle\xi, \int_{0}^{s} k(t, r) \mathrm{d} \mathscr{L}_{r}\right\rangle\right)$.

Everything we have introduced so far only relates to the probabilistic properties of Volterra-Lévy process without any details regarding its sample path behaviour. Towards the goal of proving regularity of the local time associated with $\left(z_{t}\right)_{t \in[0, T]}$, as done in Sect. 4, we require that the process $z$ is continuous in probability.

Remark 19 Recall that given a sufficiently regular Volterra kernel $k$, continuity in probability can for example be obtained from the fact $L^{p}$-convergence entails $\mathbb{P}$ convergence using, e.g. Markov's inequality. Indeed, suppose $\left(z_{t}\right)_{t \in[0, T]}$ is a VolterraLévy process, as given in Definition 17. It is readily checked that $z$ is continuous in probability if there exists a $p>0$ such that

$$
\mathbb{E}\left[\left|z_{t}-z_{s}\right|^{p}\right] \rightarrow 0 \quad \text { when } s \rightarrow t
$$

This particular fact will be used in Examples 22 and 23 below.

Below we provide three examples of different types of Volterra processes driven by Lévy noise.

Example 20 (Brownian motion) Let $\beta=2, k(t, \cdot) \in L^{2}([0, t])$ for $t \in[0, T]$. Suppose $\mathscr{L}$ is a Brownian motion with values in $\mathbb{R}^{d}$. Then, it is well known that $z_{t}=\int_{0}^{t} k(t, s) \mathrm{d} \mathscr{L}_{s}$ is well-defined in $L^{2}(\Omega)$ as a Wiener integral. The sample paths of such processes are clearly measurable, and depending on the regularity of the kernel $k$, the process may also be (Hölder) continuous. 
Example 21 (Square-integrable martingale case) Let $\beta=2, k(t, \cdot) \in L^{2}([0, t])$ for $t \in[0, T]$ and $\mathscr{L}$ be a $\left(\mathcal{F}_{t}\right)$-martingale satisfying $\mathbb{E}\left[\left|\mathscr{L}_{t}\right|^{2}\right]<\infty$, for all $t \in[0, T]$. Then, we know $z_{t}=\int_{0}^{t} k(t, s) \mathrm{d} \mathscr{L}_{s}, t \geq 0$ is well-defined according to Proposition 18 (this is also clear from classical martingale theory, e.g. [1]).

The next example considers the case of $\alpha$-stable processes and gives explicit conditions on the Volterra kernel $k$ so that the resulting Volterra process is continuous in probability.

Example 22 (Standard $\alpha$-stable case) Let $\alpha \in(0,2)$, and suppose $\mathscr{L}$ is a standard $\alpha$ stable process, as defined in Definition 13. Assume $k: \Delta_{T}^{2} \rightarrow \mathbb{R}$ is a Volterra kernel such that $k(t, \cdot) \in L^{\alpha}([0, t])$ for all $t \in[0, T]$, and that

$$
H(t, s):=\int_{0}^{s}|k(t, r)-k(s, r)|^{\alpha} \mathrm{d} r
$$

is such that $H(t, s) \rightarrow 0$ when $s \rightarrow t$ for all $t \in[0, T]$. By Proposition 18, we know that $z_{t}=\int_{0}^{t} k(t, s) \mathscr{L}_{s}, t \geq 0$ is well defined for $\alpha \in(0,2)$ (see also [27, Section 3.6 Examples]). Furthermore, $\left(z_{t}\right)_{t \in[0, T]}$ is continuous in probability. Indeed, note that $z_{t}-z_{s}=\int_{s}^{t} k(t, r) \mathrm{d} \mathscr{L}_{r}+\int_{0}^{s} k(t, r)-k(s, r) \mathrm{d} \mathscr{L}_{r}$. Using that for any $p>0$

$$
\mathbb{E}\left[\left|z_{t}-z_{s}\right|^{p}\right] \lesssim p \mathbb{E}\left[\left|\int_{s}^{t} k(t, r) \mathrm{d} \mathscr{L}_{r}\right|^{p}\right]+\mathbb{E}\left[\left|\int_{0}^{s} k(t, r)-k(s, r) \mathrm{d} \mathscr{L}_{r}\right|^{p}\right]
$$

and the relation in (3.3), there exists a $p>0$ such that

$$
\mathbb{E}\left[\left|z_{t}-z_{s}\right|^{p}\right] \lesssim_{p, \alpha, d}\left(\int_{s}^{t}|k(t, r)|^{\alpha} \mathrm{d} r\right)^{\frac{p}{\alpha}}+H(t, s)^{\frac{p}{\alpha}}
$$

where by assumption $H(t, s) \rightarrow 0$ when $s \rightarrow t$ for all $t \in[0, T]$, and the integrability of k yields out that $\int_{s}^{t}|k(t, r)|^{\alpha} \mathrm{d} r \rightarrow 0$ as $s \rightarrow t$. Therefore, we conclude by Remark 19 that $\left(z_{t}\right)_{t \in[0, T]}$ is continuous in probability.

With the above preparation at hand, we can then construct fractional $\alpha$-stable processes and give a representation of its characteristic function. We summarize this in the following example.

Example 23 (Fractional $\alpha$-stable process) Let $\mathscr{L}$ be an $\alpha$-stable process with $\alpha \in$ $(0,2]$, and consider the Volterra kernel $k(t, s)=(t-s)^{H-\frac{1}{\alpha}}, H \in(0,1)$. Then, the process $z_{t}=\int_{0}^{t} k(t, s) \mathrm{d} \mathscr{L}_{s}$ is called a fractional $\alpha$-stable process (of RiemannLiouville type) and specifically if $\alpha=2$, then $\mathscr{L}$ is a Brownian motion and $z$ is a fractional Brownian motion. Note that in this case $k(t, \cdot) \in L^{\alpha}([0, t], \mathrm{d} s)$ for any $H \in(0,1)$. There is a more detailed study of fractional processes of this type in [27, 
Chapter 7]. An application of Proposition (3.4) yields that the characteristic function associated with the fractional $\alpha$-stable process $z$ is given by

$$
\mathbb{E}\left[\exp \left(i\left\langle\xi, z_{t}\right\rangle\right)\right]=\exp \left(-c_{\alpha} \frac{|\xi|^{\alpha} t^{H \alpha}}{H \alpha}\right) .
$$

Furthermore, it is simply checked that the function $H$ defined as in (3.6) with the kernel $k(t, s)=|t-s|^{H-\frac{1}{\alpha}}$ satisfies $H(t, s) \rightarrow 0$ when $s \rightarrow t$, and thus by the same argument as used in Example 22, it is readily checked that the fractional $\alpha$-stable process $\left(z_{t}\right)_{t \in[0, T]}$ is continuous in probability.

\section{Regularity of the Local Time Associated with Volterra-Lévy Processes}

This section is devoted to prove space-time regularity of the local time associated with Volterra-Lévy processes, as defined in Sect. 3. We begin to give a notion of local non-determinism for these processes and provide a few examples of specific processes which satisfy this property.

\subsection{Local Non-determinism Condition for Volterra-Lévy Process}

The following definition of a local non-determinism condition can be seen as an extension of the concept of strong local non-determinism used in the context of Gaussian processes, see, e.g. [13,15,29].

Definition 24 Let $\mathscr{L}$ be a Lévy process with characteristic $\psi: \mathbb{R}^{d} \rightarrow \mathbb{C}$ as given in (3.1), and let $z$ be a Volterra-Lévy process (Definition 17) with Lévy process $\mathscr{L}$ and Volterra kernel $k: \Delta_{T}^{2} \rightarrow \mathbb{R}$ satisfying $k(t, \cdot) \in L^{\alpha}([0, t])$ for all $t \in[0, T]$. If for some $\zeta>0$ and $\alpha \in(0,2]$, the following inequality holds

$$
\lim _{t \downarrow 0} \inf _{s \in(0, t]} \inf _{\xi \in \mathbb{R}^{d}} \frac{\int_{s}^{t} \psi(k(t, r) \xi) \mathrm{d} r}{(t-s)^{\zeta}|\xi|^{\alpha}}>0 .
$$

Then, we say that $z$ is $(\alpha, \zeta)$-Locally non-deterministic $((\alpha, \zeta)$-LND).

Remark 25 The elementary example of a Volterra kernel is $k(t, s):=1_{[0, t]}(s)$ for any $0 \leq s \leq t \leq T$. In this case, the Volterra-Lévy process is just given as the Lévy process itself, i.e. $z_{t}=\mathscr{L}_{t}$. If we let $\mathscr{L}$ be a standard $d$-dimensional $\alpha$-stable process, condition (4.1) fulfils for $\zeta=1$. Hence, a standard $d$-dimensional $\alpha$-stable process is $(\alpha, 1)$-LND, which coincides with the conclusion in [20, Proposition 4.5, Example (a)].

Remark 26 There already exists several concepts of local non-determinism, but, as far as we know, most of them are given in terms of a condition on the variance of certain stochastic processes. The only exception we are aware of is the definition of Nolan 
in [20] for $\alpha$-stable processes, where a similar condition is stated in $L^{p}$ spaces, with $p=\alpha$ (see [20, Definition 3.3]). Of course working with general $\alpha$-stable processes, we do in general not have finite variance, and thus, the standard definitions of such a concept are not applicable. On the other hand, in the case when $\alpha=2$, we have finite variance, and then, the above criterion would be very similar to the condition for strong local non-determinism for Gaussian Volterra processes, as discussed for example in [29]. Working with the conditional characteristic function of Volterra $\alpha$ stable processes, we see, however, that this condition in some sense is what needs to be replaced in order to prove existence and regularity of local times associated with these processes.

It is readily seen that the Volterra $\alpha$-stable process satisfies (4.1), with $\zeta$ depending on the choice of kernel $k$. The condition is, however, somewhat more general, as we only require the processes to behave similarly to Volterra $\alpha$-stable processes. Let us provide an example to discuss some interesting process that satisfies the LND condition.

Example 27 (Volterra kernel) As two examples of Volterra kernel that we are interested most, we give a specific discussion here. The first one usually relates to fractional type processes, for instance, fractional Brownian motion and fractional stable processes. As we will see later, the second one makes the corresponding Volterra-Lévy process an infinitely regularising process, similarly to the Gaussian counterpart discussed in [15].

(i) For $\alpha \in(0,2], H \in(0,1)$, let $k(t, s)=F(t, s)(t-s)^{H-\frac{\alpha}{2}}$, where $F: \Delta_{T}^{2} \rightarrow$ $\mathbb{R} \backslash 0$ is continuous and $F(t, s) \simeq 1$ when $|t-s| \rightarrow 0$, where $\simeq$ means that the two sides are comparable up to a positive constant. It can be easily checked that $k(t, \cdot) \in L^{\alpha}([0, t])$ for $t \in[0, T]$.

(ii) Let $p>\frac{1}{\alpha}$, and consider the kernel $k(t):=t^{-\frac{1}{\alpha}}\left(\ln \frac{1}{t}\right)^{-p}$ for $t \in[0,1)$. It is readily seen that $k(t, \cdot) \in L^{\alpha}([0, t])$ for any $t<1$.

Example 28 (Gaussian case: $\alpha=2$ ) Let $\mathscr{L}=B$ be a Brownian motion. Then, the Gaussian Volterra process $z_{t}=\int_{0}^{t} k(t, s) \mathrm{d} B_{s}, t \in[0, T]$ is $(2, \zeta)$-LND according to definition 24 if

$$
\lim _{t \downarrow 0} \inf _{s \in(0, t]} \frac{\int_{s}^{t}|k(t, r)|^{2} \mathrm{~d} r}{(t-s)^{\zeta}}>0 .
$$

As we mentioned, the Lévy process $\mathscr{L}$ does not have to be Gaussian type processes. For non-Gaussian type $\mathscr{L}$, we mostly consider $\alpha$-stable processes or the processes which has similar behaviour to stable processes. Since the condition (4.1) only focuses on the characteristic function $\psi$ of $\mathscr{L}$, there is a large class of jump processes which can be studied here.

Example 29 (Stable type processes) Fix an $\alpha \in(0,2)$. Given a kernel $k: \Delta_{T}^{2} \rightarrow \mathbb{R}$ with $k(t, \cdot) \in L^{\alpha}([0, t])$ and satisfying for some $\zeta>0$, the following inequality

$$
\lim _{t \downarrow 0} \inf _{s \in(0, t]} \frac{\int_{s}^{t}|k(t, r)|^{\alpha} \mathrm{d} r}{(t-s)^{\zeta}}>0 .
$$


Then, the following list of processes satisfies the LND condition in Definition 24:

(i) $\mathscr{L}$ is a standard $d$-dimensional $\alpha$-stable process, i.e.

$$
\psi(\xi)=c_{\alpha}|\xi|^{\alpha}, \quad c_{\alpha}>0 .
$$

Then, obviously $z_{t}=\int_{0}^{t} k(t, r) \mathrm{d} \mathscr{L}_{r}, t>0$ is $(\alpha, \zeta)$-LND.

Besides, here if $k(t, s)=k(t-s)$ for $0 \leq s \leq t<\infty$ and $\int_{0}^{t}|k(t, s)|^{\alpha} \mathrm{d} s>$ 0 , according to Definition 24, the process $z$ is $(\alpha, 1)$-LND, which coincides the conclusion in [20, Proposition 4.5].

(ii) $\mathscr{L}=\left(\mathscr{L}_{1}, \ldots, \mathscr{L}_{d}\right)$, where $\mathscr{L}_{1}, \ldots, \mathscr{L}_{d}$ are independent 1 -dimensional standard $\alpha$-stable processes. In this case, the corresponding characteristic function $\psi$ is given by

$$
\psi(\xi)=c_{\alpha}\left(\left|\xi_{1}\right|^{\alpha}+\cdots+\left|\xi_{d}\right|^{\alpha}\right), \quad c_{\alpha}>0
$$

By Jensen's inequality, it follows that $\left|\xi_{1}\right|^{\alpha}+\ldots+\left|\xi_{d}\right|^{\alpha}=\left|\xi_{1}\right|^{2 \cdot \frac{\alpha}{2}}+\ldots+\left|\xi_{d}\right|^{2 \cdot \frac{\alpha}{2}} \geq$ $\left(\left|\xi_{1}\right|^{2}+\ldots+\left|\xi_{d}\right|^{2}\right)^{\frac{\alpha}{2}}=|\xi|^{\alpha}$ for $\alpha \in(0,2]$, which implies $\psi(\xi) \geq c_{\alpha}|\xi|^{\alpha}$. By (4.2), we conclude that $z_{t}=\int_{0}^{t} k(t, r) \mathrm{d} \mathscr{L}_{r}, t>0$, is $(\alpha, \zeta)$-LND.

(iii) $\mathscr{L}$ is a $d$-dimensional Lévy process with characteristic function

$$
\psi(\xi)=|\xi|^{\alpha} \log (2+|\xi|), \quad \xi \in \mathbb{R}^{d} .
$$

We additionally assume $\alpha \in(0,1)$ (see [17] Example 1.5). This process is not really a stable process, but the small size jumps of this process have similar behaviour to stable processes. Since $|\xi|^{\alpha} \log (2+|\xi|) \geq|\xi|^{\alpha}$ for $\xi \in \mathbb{R}^{d}$, then

$$
\lim _{t \downarrow 0} \inf _{s \in(0, t]} \inf _{\xi \in \mathbb{R}^{d}} \frac{\int_{s}^{t} \psi(k(t, r) \xi) \mathrm{d} r}{(t-s)^{\zeta}|\xi|^{\alpha}} \geq \lim _{t \downarrow 0} \inf _{s \in(0, t]} \inf _{\xi \in \mathbb{R}^{d}} \frac{\int_{s}^{t}|k(t, r)|^{\alpha} \mathrm{d} r}{(t-s)^{\zeta}}>0 .
$$

Therefore, $z_{t}=\int_{0}^{t} k(t, r) \mathrm{d} \mathscr{L}_{r}, t>0$, is $(\alpha, \zeta)$-LND.

The following theorem shows the regularity of the local time associated with Volterra-Lévy processes which is $(\alpha, \zeta)$-LND according to Definition 24.

\subsection{Regularity of the Local Time}

With the concept of local non-determinism at hand, we are now ready to prove the regularity of the local time associated with Volterra-Lévy processes, and thus also proving Theorem 1. The following theorem provides a proof of Theorem 1, as well as giving $\mathbb{P}$-a.s. bounds for the Fourier transform of the occupation measure and the local time.

Theorem 30 (Regularity of Local time) Let $z: \Omega \times[0, T] \rightarrow \mathbb{R}^{d}$ be a Lévy Volterra process with characteristic $\psi: \mathbb{R}^{d} \rightarrow \mathbb{C}$ on a complete filtered probability space $\left(\Omega, \mathcal{F},\left\{\mathcal{F}_{t}\right\}_{t \in[0, T]}, \mathbb{P}\right)$, and suppose $z$ is $(\alpha, \zeta)$-LND for some $\zeta \in\left(0, \frac{\alpha}{d}\right)$ and $\alpha \in$ 
$(0,2]$, continuous in probability, and adapted to the filtration $\left(\mathcal{F}_{t}\right)_{t \in[0, t]}$. Then, the local time $L: \Omega \times[0, T] \times \mathbb{R}^{d} \rightarrow \mathbb{R}_{+}$associated with z exists and is square integrable. Furthermore, for any $\kappa<\frac{\alpha}{2 \zeta}-\frac{d}{2}$ there exists a $\gamma>\frac{1}{2}$ such that the local time is contained in the space $\mathcal{C}_{T}^{\gamma} H^{\kappa}$.

Proof We will follow along the lines of the proof of [15, Theorem 17], but adapt to the case of Lévy processes. To this end, we will apply the stochastic sewing lemma from [19], which is provided in Lemma 37 for self-containedness.

A Fourier transform of the occupation measure $\mu_{s, t}(\mathrm{~d} x)$ yields $\int_{s}^{t} \mathrm{e}^{i\left\langle\xi, z_{r}\right\rangle} \mathrm{d} r$. Note that this coincides with the Fourier transform of the local time $L_{s, t}(x)$ whenever $L$ exists. Our first goal is therefore to show that for any $p \geq 2$, the following inequality holds for some $\lambda \geq 0$ and $\gamma \in\left(\frac{1}{2}, 1\right)$

$$
\left\|\widehat{\mu_{s, t}}(\xi)\right\|_{L^{p}(\Omega)} \lesssim\left(1+|\xi|^{2}\right)^{-\frac{\lambda}{2}}|t-s|^{\gamma}
$$

To this end, the stochastic sewing lemma (see Lemma 37) will provide us with this information. We begin to define

$$
A_{s, t}^{\xi}:=\int_{s}^{t} \mathbb{E}\left[\exp \left(i\left\langle\xi, z_{r}\right\rangle\right) \mid \mathcal{F}_{S}\right] \mathrm{d} r,
$$

and for a partition $\mathcal{P}[s, t]$ of $[s, t]$ define

$$
\mathcal{A}_{\mathcal{P}[s, t]}^{\xi}:=\sum_{u, v} A_{u, v}^{\xi}
$$

If the integrand $A^{\xi}$ satisfies the conditions (i)-(ii) in Lemma 37, then a unique limit to $\mathcal{A}_{s, t}^{\xi}=\lim _{|\mathcal{P}| \rightarrow 0} \mathcal{A}_{\mathcal{P}}^{\xi}$ exists in $L^{p}(\Omega)$. Note that then $\int_{s}^{t} \mathrm{e}^{i\left\langle\xi, z_{r}\right\rangle} \mathrm{d} r=\mathcal{A}_{s, t}^{\xi}$ in $L^{p}(\Omega)$. We continue to prove that conditions (i)-(ii) in Lemma 37 are indeed satisfied for our integrand $A$. It is already clear that $A_{s, s}^{\xi}=0$, and $A_{s, t}^{\xi}$ is $\left(\mathcal{F}_{t}\right)$-measurable. For any point $u \in[s, t]$, we define

$$
\delta_{u} f_{s, t}:=f_{s, t}-f_{s, u}-f_{u, t}
$$

for any function $f:[0, T]^{2} \rightarrow \mathbb{R}$. It follows by the tower property and linearity of conditional expectations that

$$
\begin{aligned}
\mathbb{E}\left[\delta_{u} A_{s, t}^{\xi} \mid \mathcal{F}_{s}\right]= & \mathbb{E}\left[\int_{s}^{t} \mathbb{E}\left[\exp \left(i\left\langle\xi, z_{r}\right\rangle\right) \mid \mathcal{F}_{s}\right] \mathrm{d} r\right. \\
& \left.-\int_{s}^{t} \mathbb{E}\left[\exp \left(i\left\langle\xi, z_{r}\right\rangle\right) \mid \mathcal{F}_{s}\right] \mathrm{d} r-\int_{s}^{u} \mathbb{E}\left[\exp \left(i\left\langle\xi, z_{r}\right\rangle\right) \mid \mathcal{F}_{u}\right] \mathrm{d} r \mid \mathcal{F}_{S}\right]=0 .
\end{aligned}
$$


At last, we will need to control the term $\left\|\delta_{u} A_{s, t}^{\xi}\right\|_{L^{p}(\Omega)}$. To this end, using Proposition 18 , we know that

$$
A_{s, t}^{\xi}=\int_{s}^{t} \mathcal{E}_{0, s, r}(\xi) \exp \left(-\int_{s}^{r} \psi(k(r, l) \xi) \mathrm{d} l\right) \mathrm{d} r
$$

where $\mathcal{E}$ is defined as in (3.5). Therefore, it is readily checked that

$$
\begin{aligned}
\delta_{u} A_{s, t}^{\xi}= & \int_{u}^{t} \mathcal{E}_{0, s, r}(\xi) \exp \left(-\int_{s}^{r} \psi(k(r, l) \xi) \mathrm{d} l\right) \\
& -\mathcal{E}_{0, u, r}(\xi) \exp \left(-\int_{u}^{r} \psi(k(r, l) \xi) \mathrm{d} l\right) \mathrm{d} r .
\end{aligned}
$$

Of course, moments of the complex exponential $\mathcal{E}_{0, s, r}(\xi)$ is bounded by 1 , i.e. for any $r \in[s, t],\left\|\mathcal{E}_{0, s, r}(\xi)\right\|_{L^{p}(\Omega)} \leq 1$, and therefore, it follows that

$$
\left\|\delta_{u} A_{S, t}^{\xi}\right\|_{L^{p}(\Omega)} \lesssim \int_{u}^{t} \exp \left(-\int_{s}^{r} \psi(k(r, l) \xi) \mathrm{d} l\right)+\exp \left(-\int_{u}^{r} \psi(k(r, l) \xi) \mathrm{d} l\right) \mathrm{d} r .
$$

Using the fact that $z$ is $(\alpha, \zeta)$-LND for some $\zeta \in(0,1)$, and using that $(r-s)^{\zeta} \geq$ $(r-u)^{\zeta}$ we obtain the estimate

$$
\left\|\delta_{u} A_{s, t}^{\xi}\right\|_{L^{p}(\Omega)} \lesssim \int_{u}^{t} \exp \left(-c|\xi|^{\alpha}(r-u)^{\zeta}\right) \mathrm{d} r
$$

Note in particular that this holds for any $p \geq 2$. By the property of the exponential function, we have that for any $\eta \in \mathbb{R}_{+}$

$$
\exp \left(-c_{\alpha}|\xi|^{\alpha}(r-u)^{\zeta}\right) \leq \exp \left(T^{\zeta}\right)\left(1+|\xi|^{\alpha}\right)^{-\eta}(r-u)^{-\zeta \eta} \sup _{q \in \mathbb{R}_{+}} q^{\eta} \exp (-q)
$$

Since $1+|\xi|^{\alpha} \lesssim\left(1+|\xi|^{2}\right)^{\frac{\alpha}{2}}$ for all $\alpha \in(0,2]$, applying this relation in (4.3), and assuming that $0 \leq \eta \zeta<\frac{1}{2}$, it follows that for all $p \geq 2$ there exists a $\gamma>\frac{1}{2}$ and $\lambda<\frac{\alpha}{2 \zeta}$

$$
\left\|\delta_{u} A_{s, t}^{\xi}\right\|_{L^{p}(\Omega)} \lesssim\left(1+|\xi|^{2}\right)^{-\frac{\lambda}{2}}(t-u)^{\gamma}
$$

Thus, both conditions of (A.1) in Lemma 37 are satisfied. By a simple addition and subtraction of the integrand $A_{s, t}^{\xi}$ in (A.2), it follows that for all $p \geq 2$ the limiting 
process $\mathcal{A}_{s, t}^{\xi}$ satisfies

$$
\left\|\mathcal{A}_{s, t}^{\xi}\right\|_{L^{p}(\Omega)} \lesssim\left(1+|\xi|^{2}\right)^{-\frac{\lambda}{2}}(t-u)^{\gamma}
$$

We will now show that $\widehat{\mu_{s, t}}(\xi)=\mathcal{A}_{s, t}^{\xi}$ in $L^{p}(\Omega)$. For a partition $\mathcal{P}$ of $[s, t]$, we have

$$
\left\|\widehat{\mu_{s, t}}(\xi)-\mathcal{A}_{s, t}^{\xi}\right\|_{L^{p}(\Omega)} \leq \sum_{[u, v] \in \mathcal{P}} \int_{u}^{v}\left\|\mathrm{e}^{i\left\langle\xi, z_{r}\right\rangle}-\mathbb{E}\left[\mathrm{e}^{i\left\langle\xi, z_{r}\right\rangle} \mid \mathcal{F}_{u}\right]\right\|_{L^{p}(\Omega)} \mathrm{d} r .
$$

By Minkowski's inequality, we have

$$
\begin{aligned}
& \left\|\mathrm{e}^{i\left\langle\xi, z_{r}\right\rangle}-\mathbb{E}\left[\mathrm{e}^{i\left\langle\xi, z_{r}\right\rangle} \mid \mathcal{F}_{u}\right]\right\|_{L^{p}(\Omega)} \\
& \quad \leq\left\|\mathrm{e}^{i\left\langle\xi, z_{r}\right\rangle}-\mathrm{e}^{i\left\langle\xi, z_{u}\right\rangle}\right\|_{L^{p}(\Omega)}+\left\|\mathbb{E}\left[\mathrm{e}^{i\left\langle\xi, z_{r}\right\rangle}-\mathrm{e}^{i\left\langle\xi, z_{u}\right\rangle} \mid \mathcal{F}_{u}\right]\right\|_{L^{p}(\Omega)},
\end{aligned}
$$

and by Jensen's inequality it follows that

$$
\left\|\mathbb{E}\left[\mathrm{e}^{i\left\langle\xi, z_{r}\right\rangle}-\mathrm{e}^{i\left\langle\xi, z_{u}\right\rangle} \mid \mathcal{F}_{u}\right]\right\|_{L^{p}(\Omega)} \leq\left\|\mathrm{e}^{i\left\langle\xi, z_{r}\right\rangle}-\mathrm{e}^{i\left\langle\xi, z_{u}\right\rangle}\right\|_{L^{p}(\Omega)}
$$

This implies that

$$
\left\|\mathrm{e}^{i\left\langle\xi, z_{r}\right\rangle}-\mathbb{E}\left[\mathrm{e}^{i\left\langle\xi, z_{r}\right\rangle} \mid \mathcal{F}_{u}\right]\right\|_{L^{p}} \leq 2\left\|\mathrm{e}^{i\left\langle\xi, z_{r}\right\rangle}-\mathrm{e}^{i\left\langle\xi, z_{u}\right\rangle} \mid \mathcal{F}_{u}\right\|_{L^{p}(\Omega)}
$$

Furthermore, for fixed $\epsilon>0$ then

$$
\begin{aligned}
\left\|\mathrm{e}^{i\left\langle\xi, z_{r}\right\rangle}-\mathrm{e}^{i\left\langle\xi, z_{u}\right\rangle}\right\|_{L^{p}(\Omega)} & \leq\left\|\left(\mathrm{e}^{i\left\langle\xi, z_{r}\right\rangle}-\mathrm{e}^{i\left\langle\xi, z_{u}\right\rangle}\right) 1_{\left|\mathrm{e}^{i\left\langle\xi, z_{r}\right\rangle}-\mathrm{e}^{i\left\langle\xi, z_{u}\right\rangle}\right|>\epsilon}\right\|_{L^{p}(\Omega)}+\epsilon \\
& \leq \mathbb{P}\left(\left|\mathrm{e}^{i\left\langle\xi, z_{r}\right\rangle}-\mathrm{e}^{i\left\langle\xi, z_{u}\right\rangle}\right|>\epsilon\right)+\epsilon
\end{aligned}
$$

since $\left\|\mathrm{e}^{i\left\langle\xi, z_{r}\right\rangle}-\mathrm{e}^{i\left\langle\xi, z_{u}\right\rangle}\right\|_{L^{q}(\Omega)} \leq 1$ for any $q$. We conclude that for any $\epsilon>0$

$$
\left\|\widehat{\mu_{s, t}}(\xi)-\mathcal{A}_{s, t}^{\xi}\right\|_{L^{p}(\Omega)} \leq 2 \epsilon(t-s)+2 \sum_{[u, v] \in \mathcal{P}} \int_{u}^{v} \mathbb{P}\left(\left|\mathrm{e}^{i\left\langle\xi, z_{r}\right\rangle}-\mathrm{e}^{i\left\langle\xi, z_{u}\right\rangle}\right|>\epsilon\right) \mathrm{d} r .
$$

Since this holds for any partition $\mathcal{P}$, letting the mesh tend to 0 , and using the assumption that $z$ is continuous in probability, it follows that $\mathrm{e}^{i\left\langle\xi, z_{r}\right\rangle}$ is continuous in probability (see, e.g. the continuous mapping theorem), and thus

$$
\begin{aligned}
& \lim _{|\mathcal{P}| \rightarrow 0} \sum_{[u, v] \in \mathcal{P}} \int_{u}^{v} \mathbb{P}\left(\left|\mathrm{e}^{i\left\langle\xi, z_{r}\right\rangle}-\mathrm{e}^{i\left\langle\xi, z_{u}\right\rangle}\right|>\epsilon\right) \mathrm{d} r \\
& \leq \lim _{|\mathcal{P}| \rightarrow 0} \sup _{[u, v] \in \mathcal{P}} \sup _{r \in[u, v]} \mathbb{P}\left(\left|\mathrm{e}^{i\left\langle\xi, z_{r}\right\rangle}-\mathrm{e}^{i\left\langle\xi, z_{u}\right\rangle}\right|>\epsilon\right)(t-s)=0 .
\end{aligned}
$$


Since (4.5) holds for any partition (also for partitions with infinitesimal mesh), we conclude that for any $\epsilon>0$

$$
\left\|\widehat{\mu_{s, t}}(\xi)-\mathcal{A}_{s, t}^{\xi}\right\|_{L^{p}(\Omega)} \leq 2 \epsilon(t-s)
$$

and since $\epsilon$ can be chosen arbitrarily small, we conclude that $\widehat{\mu_{s, t}}(\xi)=\mathcal{A}_{s, t}^{\xi}$ in $L^{p}(\Omega)$.

We move on to estimate the Sobolev norm $\left\|L_{s, t}\right\|_{H^{\kappa}}$ for some appropriate $\kappa \in \mathbb{R}$. We begin to observe that

$$
\|\| \mu_{s, t}\left\|_{H^{\kappa}}\right\|_{L^{p}(\Omega)}=\left[\mathbb{E}\left(\left.\int_{\mathbb{R}^{d}}\left(1+|\xi|^{2}\right)^{\kappa} \sqrt{\mu_{s, t}}(\xi)\right|^{2} d \xi\right)^{\frac{p}{2}}\right]^{\frac{1}{p}}
$$

By Minkowski’s inequality, it follows that

$$
\|\| \mu_{s, t}\left\|_{H^{\kappa}}\right\|_{L^{p}(\Omega)} \lesssim\left\|\left(1+|\cdot|^{2}\right)^{\frac{\kappa}{2}}\right\| \widehat{\mu_{s, t}}\left\|_{L^{p}(\Omega)}\right\|_{L^{2}\left(\mathbb{R}^{d}\right)}
$$

and then use the bound from (4.4) to observe that

$$
\|\| \mu_{s, t}\left\|_{H^{\kappa}}\right\|_{L^{p}(\Omega)} \lesssim(t-s)^{\gamma}\left\|\left(1+|\cdot|^{2}\right)^{\frac{\kappa-\lambda}{2}}\right\|_{L^{2}(\mathbb{R})}
$$

Choosing $\kappa=\lambda-\frac{d}{2}-\epsilon$ for some arbitrarily small $\epsilon>0$, it follows that

$$
\left\|\left(1+|\cdot|^{2}\right)^{\frac{\kappa-\lambda}{2}}\right\|_{L^{2}(\mathbb{R})}=\left\|\left(1+|\cdot|^{2}\right)^{\frac{d}{4}-\epsilon / 2}\right\|_{L^{2}(\mathbb{R})}<\infty .
$$

Recalling that $\lambda<\frac{\alpha}{2 \zeta}$, since $\epsilon>0$ could be chosen arbitrarily small, we obtain that for any $\kappa<\frac{\alpha}{2 \zeta}-\frac{d}{2}$ there exists a $\gamma>\frac{1}{2}$ such that

$$
\|\| \mu_{s, t}\left\|_{H^{\kappa}}\right\|_{L^{p}(\Omega)} \lesssim(t-s)^{\gamma} .
$$

Since $p \geq 2$ can be chosen arbitrarily large, we conclude by Kolmogorov's theorem of continuity that there exists a set $\Omega^{\prime} \subset \Omega$ of full measure such that for all $\omega \in \Omega^{\prime}$ there exists a $C(\omega)>0$ such that

$$
\left\|\mu_{s, t}(\omega)\right\|_{H^{\kappa}} \leq C(\omega)(t-s)^{\gamma}
$$

In particular, this implies that for almost all $\omega \in \Omega, \mu(\omega) \in L^{2}\left(\mathbb{R}^{d}\right)$ and thus the local time $L(\omega)$ (given as the density of $\mu$ ) exists, and our claim follows.

Remark 31 In the case, when $\alpha=2$, then $X$ is a Gaussian process, and Theorem 30 provides the same regularity of the associated local time for a Gaussian Volterra process as proven in for example [15] (or without considering the joint time regularity, as shown in $[5,14,21])$. This theorem can therefore be seen as an extension of this work to the class of Volterra-Lévy processes. 
We will now give several examples on the application of Theorem 30 to show the regularity of the local time for a few specific Volterra $\alpha$-stable processes. All of the following examples also were studied for dimension $d=1$ in [20, Corollary 4.6, Examples], it shows that the local time $L_{t}(x)$ of a Volterra $\alpha$-stable process exists a.s. and is continuous for $(t, x) \in[0, T] \times \mathbb{R}$, furthermore for fixed $t \in[0, T], L_{t}(x)$ is Hölder continuous for $x \in \mathbb{R}$ with some order less than 1 . The method therein [20] heavily relies on the $L^{\alpha}$-representation for $\alpha$-stable processes.

Example 32 (Regularity of the local time for Volterra $\alpha$-stable processes) We consider Volterra- $\alpha$-stable processes

$$
z_{t}=\int_{0}^{t} k(t-s) \mathrm{d} \mathscr{L}_{s}, \quad t \geq 0,
$$

where $\mathscr{L}$ is a $d$-dimensional standard $\alpha$-stable process with $\alpha \in(0,2]$.

(i). Let $d=1$ and $k(t) \equiv 1$ for all $t \geq 0$, then

$$
z_{t}=\mathscr{L}_{t}
$$

is an one-dimensional standard $\alpha$-stable process. When $\alpha \in(1,2]$, we know that an one-dimensional standard $\alpha$-stable process $\mathscr{L}$ is $(\alpha, 1)$-LND, and continuous in probability. According to above theorem, there exists a $\gamma>\frac{1}{2}$, such that the local time associated with $z$, and thus also $\mathscr{L}$ is contained in $\mathcal{C}_{T}^{\gamma} H^{\kappa}$ for any $\kappa<\frac{\alpha}{2}-\frac{1}{2}$, P-a.s..

(ii) Let $k(t)=\mathrm{e}^{-a t}$ with $a>0$. Then, the Ornstein-Uhlenbeck-Lévy process

$$
z_{t}=\int_{0}^{t} \mathrm{e}^{-a(t-s)} \mathrm{d} \mathscr{L}_{s}, \quad t \geq 0
$$

is $(\alpha, \zeta)$-LND for $\alpha \in(0,2]$ and $\zeta=1$, and continuous in probability. Hence, there exists a $\gamma>\frac{1}{2}$, such that the process $z$ has a local time $L \in \mathcal{C}_{T}^{\gamma} H^{\kappa}$ for any $\kappa<\frac{\alpha}{2}-\frac{d}{2}, \mathbb{P}$-a.s..

(iii) Let $\left(z_{t}\right)_{t \in[0, T]}$ be a fractional $\alpha$-stable process as in Example 23. Then, $\left(z_{t}\right)_{t \in[0, T]}$ is continuous in probability, and there exists a $\gamma>\frac{1}{2}$ such that the local time $L$ associated with $z$ is contained in $\mathcal{C}_{T}^{\gamma} H^{\kappa}$ for any $\kappa<\frac{1}{2 H}-\frac{d}{2}, \mathbb{P}$-a.s.. Note that in this case, one obtains the same regularity for the local time, as one would for the fractional Brownian motion (see, e.g. [15]).

(iv) Fix $T<1$ and let $k(t)=t^{-\frac{1}{\alpha}} \ln \left(\frac{1}{t}\right)^{-p}$ for some $p>1$, and suppose $\left(\mathscr{L}_{t}\right)_{t \in[0, T]}$ is a standard $\alpha$-stable process for some $\alpha \in(0,2]$. Let $\left(z_{t}\right)_{t \in[0, T]}$ be the Volterra-Lévy process built from $k$ and $\mathscr{L}$. It is readily checked with, Example 22 and Example 27 that this process is continuous in probability. Moreover, note that in this case $z$ is $(\alpha, \zeta)$-LND for any $\zeta>0$. Thus, for any $\gamma \in(0,1)$ the local time $L$ associated with $z$ is contained in $\mathcal{C}_{T}^{\gamma} H^{\kappa}$ for any $\kappa \in \mathbb{R}, \mathbb{P}$-a.s.. Furthermore, if $z$ is càdlàg, then the 
local time $L$ has compact support, $\mathbb{P}$-a.s. and thus $L \in \mathcal{C}^{\gamma}\left([0, T] ; \mathcal{D}\left(\mathbb{R}^{d}\right)\right), \mathbb{P}$-a.s. where $\mathcal{D}\left(\mathbb{R}^{d}\right)$ denotes the space of test functions on $\mathbb{R}^{d}$. This proves in particular Corollary 2.

\section{Regularization of ODEs Perturbed by Volterra-Lévy Processes}

With the knowledge of the spatio-temporal regularity of the local time associated with a Volterra-Lévy process, we can solve additive SDEs with possibly distributionalvalued drift's coefficients. The goal of this section is to prove Theorem 4. To this end, we will recall some of the tools from the theory of nonlinear Young integrals and corresponding equations. This theory for construction of integrals and equations is by now well known (see, e.g. $[6,16]$ and more recently $[12,15]$ for an overview), but for the sake of self-containedness, we have included some short versions of proofs in the appendix. We also mention that conditions for existence and uniqueness of nonlinear Young equations can be stated in more general terms than what is used here. We choose to work with a simple set of conditions to provide a clear picture of the regularising effect in SDEs driven by Volterra-Lévy noise, in contrast to the full generality which could be accessible. More general conditions for existence and uniqueness of nonlinear Young equations can for example be found in [12].

Lemma 33 Suppose $\Gamma:[0, T] \times \mathbb{R}^{d} \rightarrow \mathbb{R}^{d}$ is contained in $\mathcal{C}_{T}^{\gamma} \mathcal{C}^{\kappa}$ for some $\gamma \in\left(\frac{1}{2}, 1\right)$ and $\kappa>\frac{1}{\gamma}$, and satisfies the following inequalities for $(s, t) \in \Delta_{2}^{T}$ and $\xi, \tilde{\xi} \in \mathbb{R}^{d}$

$$
\begin{aligned}
\text { (i) } & \left|\Gamma_{s, t}(\xi)\right|+\left|\nabla \Gamma_{s, t}(\xi)\right| \lesssim|t-s|^{\gamma} \\
\text { (ii) } & \left|\Gamma_{s, t}(\xi)-\Gamma_{s, t}(\tilde{\xi})\right| \lesssim|t-s|^{\gamma}|\xi-\tilde{\xi}| \\
\text { (iii) } & \left|\nabla \Gamma_{s, t}(\xi)-\nabla \Gamma_{s, t}(\tilde{\xi})\right| \lesssim|t-s|^{\gamma}|\xi-\tilde{\xi}|^{\kappa-1} .
\end{aligned}
$$

Then, for any $\xi \in \mathbb{R}^{d}$ there exists a unique solution to the equation

$$
y_{t}=\xi+\int_{0}^{t} \Gamma_{\mathrm{d} r}\left(y_{r}\right) .
$$

Here, the integral is interpreted as the nonlinear Young integral described in Appendix $B$

$$
\int_{0}^{t} \Gamma_{\mathrm{d} r}\left(y_{r}\right)=\lim _{|\mathcal{P}| \rightarrow 0} \sum_{[u, v] \in \mathcal{P}} \Gamma_{u, v}\left(y_{u}\right),
$$

for any partition $\mathcal{P}$ of $[0, t]$.

Proof See proof in Appendix B. 
From here on, all analysis is done pathwise. That is, we now consider a subset $\Omega^{\prime} \subset \Omega$ of full measure such that for all $\omega \in \Omega^{\prime}$ the local time $L(\omega)$ associated with a Volterra-Lévy process is contained in $\mathcal{C}_{T}^{\gamma} H^{\kappa}$, for $\gamma$ and $\kappa$ as given through Theorem 30. With a slight abuse of notation, we will write $L=L(\omega)$.

Before moving on to prove existence and uniqueness of ODEs perturbed by Volterra-Lévy processes, we will need a technical proposition on the convolution of the local time with certain (possibly distributional) vector fields.

Proposition 34 Let $\left(z_{t}\right)_{t \in[0, T]}$ be a Volterra-Lévy process which is continuous in probability and $(\alpha, \zeta)-L N D$ for some $\zeta \in(0,1]$, such that the associated local time $L$ is contained in $\mathcal{C}_{T}^{\gamma} H^{\kappa}$ for some $\gamma>\frac{1}{2}$ and $\kappa<\frac{\alpha}{2 \zeta}-\frac{d}{2}$. Suppose $b \in H^{\beta}$ for some $\beta \in \mathbb{R}$. Then, the following inequality holds for any $\theta<\beta+\kappa$ and $(s, t) \in \Delta_{T}^{2}$

$$
\left\|b * \bar{L}_{s, t}\right\|_{\mathcal{C}^{\theta}} \lesssim\|b\|_{H^{\beta}}\|L\|_{\mathcal{C}_{T}^{\gamma} H^{\kappa}}|t-s|^{\gamma}, \quad \mathbb{P}-\text { a.s. }
$$

Here, $\bar{L}_{t}(x)=L_{t}(-x)$.

Proof From Theorem 30, we know that $L_{s, t} \in H^{\kappa}$ for $\kappa<\frac{\alpha}{2 \zeta}-\frac{d}{2}$, thus, an application of Young's convolution inequality reveals that (5.3) holds.

A combination of Lemma 33 and Proposition 34 provides the existence and uniqueness of ODEs perturbed by $(\alpha, \zeta)$-LND Volterra-Lévy processes. The following corollary and proposition can be seen as proof of Theorem 4.

Corollary 35 (SDEs driven by stable Volterra processes) Let $\left(z_{t}\right)_{t \in[0, T]}$ be a VolterraLévy process which is continuous in probability, and $(\alpha, \zeta)-L N D$ according to definition 24 for some $\zeta \in\left(0, \frac{\alpha}{d}\right)$ and $\alpha \in(0,2]$. Suppose $b \in H^{\beta}$ for some $\beta \in \mathbb{R}$ such that the following inequality holds $\beta+\frac{\alpha}{2 \zeta}-\frac{d}{2} \geq 2$. Then, for any $\xi \in \mathbb{R}^{d}$ there exists a unique solution $y \in \mathcal{C}_{T}^{\gamma}\left(\mathbb{R}^{d}\right)$ to the equation

$$
y_{t}=\xi+\int_{0}^{t} b * \bar{L}_{\mathrm{d} r}\left(y_{r}\right), \quad t \in[0, T]
$$

Here, the integral and solution is interpreted pathwise in sense of Lemma 33 by setting $\Gamma_{s, t}(x):=b * \bar{L}_{s, t}(x)$, where we recall that $\bar{L}_{t}(x)=L_{t}(-x)$ and $L$ is the local time

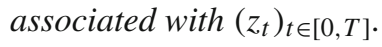

Proof By Proposition 34, we know that $b * L \in \mathcal{C}_{T}^{\gamma} \mathcal{C}^{\theta}$ for any $\theta<\beta+\frac{\alpha}{2 \zeta}-\frac{d}{2}$. Since $\beta+\frac{\alpha}{2 \zeta}-\frac{d}{2} \geq 2$, set $\Gamma_{s, t}(x):=b * \bar{L}_{s, t}(x)$, and it follows directly that conditions (i)-(iii) of Lemma 33 are satisfied, and thus, a unique solution to (5.4) exists.

Additionally to existence and uniqueness, the authors of [15] provided a general program to prove higher order differentiability of the flow mapping $\xi \mapsto y_{t}(\xi)$. We will here apply this program in order to show differentiability of flows associated with 
ODEs perturbed by sample paths of a Volterra-Lévy process. It is well known that if $b \in C^{k}$ for some $k \geq 1$, the flow $\xi \mapsto y_{t}(\xi)$ where $y$ is the solution to the ODE

$$
y_{t}=\xi+\int_{0}^{t} b\left(y_{r}\right) \mathrm{d} r
$$

is $k$-times differentiable. Translating this to the abstract framework of nonlinear Young equations; let $y$ be the solution to

$$
y_{t}=\xi+\int_{0}^{t} \Gamma_{\mathrm{d} r}\left(y_{r}\right), \quad \xi \in \mathbb{R}^{d}
$$

where $\Gamma \in \mathcal{C}_{T}^{\gamma} \mathcal{C}^{\kappa}$ for some $\kappa>\frac{1}{\gamma}$. Then, the flow $\xi \mapsto y_{t}(\xi)$ is $\kappa$ times differentiable. Recall that $\Gamma$ in our setting represents the convolution between the (possibly distributional) vector field $b$ and the local time associated with the irregular path of a Volterra-Lévy process. We therefore provide a proposition to highlight the relationship between the regularity of the vector field $b$, the regularity of the local time associated with a Volterra-Lévy process, and the differentiability of the flow.

Proposition 36 Let $\left(z_{t}\right)_{t \in[0, T]}$ be a Volterra-Lévy process taking values in $\mathbb{R}^{d}$ which is continuous in probability, and $(\alpha, \zeta)$-LND for some $\alpha \in(0,2]$ and $\zeta \in\left(0, \frac{\alpha}{d}\right)$. Suppose $b \in H^{\beta}$ for some $\beta \in \mathbb{R}$ such that $\beta+\frac{\alpha}{2 \zeta}-\frac{d}{2} \geq 1+n$ for some integer $n \geq 1$. Let $y(\xi) \in \mathcal{C}_{T}^{\gamma}\left(\mathbb{R}^{d}\right)$ denote the solution to (5.4) starting in $\xi \in \mathbb{R}^{d}$. Then, the flow map $\xi \mapsto y$. $(\xi)$ is n-times Fréchet differentiable.

Proof This result for abstract Young equations was proven in [15, Thm. 2], but we give a short outline of the proof here. Denote by $\theta=\beta+\frac{\alpha}{2 \zeta}-\frac{d}{2}$, and since $\theta \geq 2$ it follows that there exists a unique solution to (5.4). We will prove the differentiability of the flow by induction and begin to show the existence of the first derivative. It is readily checked that the first derivative of the flow $\xi \mapsto y$. $(\xi)$ needs to satisfy the equation

$$
\nabla y_{t}(\xi)=1+\int_{0}^{t} \nabla \Gamma_{\mathrm{d} r}\left(y_{r}(\xi)\right) \nabla y_{r}(\xi), \quad \text { for } \quad t \in[0, T]
$$

where the integral is understood in sense of the nonlinear Young integral in Lemma 38,by setting $\Gamma_{s, t}^{1}\left(u_{s}\right)=\nabla \Gamma_{s, t}\left(y_{s}(\xi)\right) u_{s}$ where $u_{s}=\nabla y_{s}(\xi) \in \mathbb{R}^{d \times d}$. Since (5.5) is a linear equation, existence and uniqueness can be simply verified following along the lines of the proof of Lemma 33.

Acknowledgements F.A. Harang gratefully acknowledges financial support from the STORM project 274410, funded by the Research Council of Norway. Financial support for C. Ling by the DFG through the CRC 1283 "Taming uncertainty and profiting from randomness and low regularity in analysis, stochastics and their applications" is acknowledged. 
Funding Open Access funding enabled and organized by Projekt DEAL.

Open Access This article is licensed under a Creative Commons Attribution 4.0 International License, which permits use, sharing, adaptation, distribution and reproduction in any medium or format, as long as you give appropriate credit to the original author(s) and the source, provide a link to the Creative Commons licence, and indicate if changes were made. The images or other third party material in this article are included in the article's Creative Commons licence, unless indicated otherwise in a credit line to the material. If material is not included in the article's Creative Commons licence and your intended use is not permitted by statutory regulation or exceeds the permitted use, you will need to obtain permission directly from the copyright holder. To view a copy of this licence, visit http://creativecommons.org/licenses/by/4.0/.

\section{Appendix A. Stochastic Sewing Lemma}

We recite the stochastic Sewing lemma given in [19] for self containedness. However, we refer to the aforementioned article for a discussion and full proof of this statement.

Lemma 37 Let $\left(\Omega, \mathcal{F},\left\{\mathcal{F}_{t}\right\}_{t \in[0, T]}, \mathbb{P}\right)$ be a complete probability space, where $\mathcal{F}_{0}$ contains all $\mathbb{P}$-null sets. Suppose $p \geq 2$ and let $A: \Delta_{T}^{2} \rightarrow \mathbb{R}^{d}$ be a stochastic process such that $A_{s, s}=0, A_{s, t}$ is $\left(\mathcal{F}_{t}\right)$-measurable, and $(s, t) \mapsto A_{s, t}$ is right-continuous from $\Delta_{T}^{2}$ into $L^{p}(\Omega)$. Set $\delta_{u} A_{s, t}:=A_{s, t}-A_{s, u}-A_{u, t}$ for all $(s, u, t) \in \Delta_{T}^{3}$ and assume that there exists constants $\beta>1, \kappa>\frac{1}{2}$, and $C_{1}, C_{2}>0$ such that

$$
\begin{aligned}
\left\|\mathbb{E}\left[\delta_{u} A_{s, t} \mid \mathcal{F}_{s}\right]\right\|_{L^{p}(\Omega)} & \leq C_{1}|t-s|^{\beta} \\
\left\|\delta_{u} A_{s, t}\right\|_{L^{p}(\Omega)} & \leq C_{2}|t-s|^{\kappa} .
\end{aligned}
$$

Then, there exists a unique (up to modifications) $\left(\mathcal{F}_{t}\right)$-adapted stochastic process $\mathcal{A}$ such that the following properties are satisfied:

(i) $\mathcal{A}:[0, T] \rightarrow L^{p}(\Omega)$ is right continuous, and $\mathcal{A}_{0}=0$.

(ii) There exists two constants $C_{1}, C_{2}>0$ such that the following inequalities hold

$$
\begin{aligned}
\left\|\mathcal{A}_{s, t}-A_{s, t}\right\|_{L^{p}(\Omega)} & \leq C_{1}|t-s|^{\beta}+C_{2}|t-s|^{\kappa} \\
\left\|\mathbb{E}\left[\mathcal{A}_{s, t}-A_{s, t} \mid \mathcal{F}_{s}\right]\right\|_{L^{p}(\Omega)} & \leq C_{1}|t-s|^{\beta},
\end{aligned}
$$

where we write $\mathcal{A}_{s, t}=\mathcal{A}_{t}-\mathcal{A}_{s}$.

Furthermore, for every $(s, t) \in \Delta_{T}^{2}$ and partition $\mathcal{P}$ of $[s, t]$, define

$$
A_{s, t}^{\mathcal{P}}:=\sum_{[u, v] \in \mathcal{P}} A_{u, v} .
$$

Then, $A_{s, t}^{\mathcal{P}}$ converge to $\mathcal{A}_{s, t}$ in $L^{p}(\Omega)$ when the mesh size $|\mathcal{P}| \rightarrow 0$. 


\section{Appendix B. Nonlinear Young integration and equations}

This section is devoted to give the necessary background regarding the nonlinear young integral, and Young equations. We begin to prove the existence of the nonlinear Young integral.

Lemma 38 Let $\Gamma:[0, T] \times \mathbb{R}^{d} \rightarrow \mathbb{R}^{d}$ be contained in $\mathcal{C}_{T}^{\gamma} \mathcal{C}^{1}$ and satisfy the following condition for $x, y \in \mathbb{R}^{d}$

$$
\left|\Gamma_{s, t}(x)\right| \lesssim\|\Gamma\|_{\mathcal{C}_{T}^{\gamma} \mathcal{C}^{1}}|t-s|^{\gamma} \quad \text { and } \quad\left|\Gamma_{s, t}(x)-\Gamma_{s, t}(y)\right| \lesssim\|\Gamma\|_{\mathcal{C}_{T}^{\gamma} \mathcal{C}^{1}}|x-y \| t-s|^{\gamma}
$$

for some $\gamma \in(0,1)$. Furthermore, suppose $y:[0, T] \rightarrow \mathbb{R}^{d}$ is contained in $\mathcal{C}_{T}^{\eta}$ such that $\gamma+\eta>1$. For a partition $\mathcal{P}$ of the interval $[0, T]$, define $\Xi_{s, t}:=\Gamma_{s, t}\left(y_{s}\right)$ and the sum

$$
\mathcal{I}_{\mathcal{P}}=\sum_{[u, v] \in \mathcal{P}} \Xi_{u, v}
$$

Then, there exists a unique function $\mathcal{I} \in \mathcal{C}_{T}^{\gamma}$ satisfying $\mathcal{I}_{s, t}=\mathcal{I}_{t}-\mathcal{I}_{s}$ given by $\mathcal{I}_{t}:=\lim _{|\mathcal{P}| \rightarrow 0} \mathcal{I}_{\mathcal{P}}$. We then define

$$
\int_{s}^{t} \Gamma_{\mathrm{d} r}\left(y_{r}\right)=\mathcal{I}_{s, t}
$$

Moreover, we have that $\|\delta \Gamma(y)\|_{\mathcal{C}^{\gamma+\eta}} \lesssim\|\Gamma\|_{\mathcal{C}_{T}^{\gamma} \mathcal{C}^{1}}\|y\|_{\mathcal{C}^{\eta}}$, and it follows from [11, Lemma 4.2] that

$$
\left|\int_{s}^{t} \Gamma_{\mathrm{d} r}\left(y_{r}\right)-\Gamma_{s, t}\left(y_{s}\right)\right| \lesssim\|\Gamma\|_{\mathcal{C}_{T}^{\gamma} \mathcal{C}^{1}}\|y\|_{\mathcal{C}^{\eta}|t-s|^{\eta+\gamma} .}
$$

Proof To prove this, we make use of the classical sewing lemma from the theory of rough paths (see [11, Lemma 4.2]). Set $\Xi_{s, t}=\Gamma_{s, t}\left(y_{s}\right)$. Then, we know from the sewing lemma that the abstract Riemann sum in (B.2) converge and (B.3) holds if there exists a $\beta>1$, such that

$$
\left|\Xi_{s, t}\right| \lesssim|t-s|^{\gamma} \quad\left|\delta_{u} \Xi_{s, t}\right| \lesssim|t-s|^{\beta}
$$

where $\delta_{u} \Xi_{s, t}=\Xi_{s, t}-\Xi_{s, u}-\Xi_{u, t}$. It follows by elementary algebraic manipulations that

$$
\delta_{u} \Xi_{s, t}=\Gamma_{u, t}\left(y_{s}\right)-\Gamma_{u, t}\left(y_{u}\right),
$$


and thus invoking the assumption in (B.1), we obtain that

$$
\left|\delta_{u} \Xi_{s, t}\right| \lesssim|t-u|^{\gamma}\left|y_{s}-y_{u}\right| \lesssim|t-s|^{\gamma+\eta}
$$

Since $\Gamma \in \mathcal{C}_{T}^{\gamma}$, and due to the assumption that $\eta+\gamma>1$, it follows that (B.4) is satisfied, and thus our claim follows from the sewing lemma ([11, Lemma 4.2]).

Remark 39 Of course, the nonlinear Young integral coincides with the classical Young integral if the abstract integrand $\Gamma_{s, t}\left(y_{s}\right)$ is for example given by $\Gamma_{s, t}\left(y_{s}\right)=y_{s} X_{s, t}$, for some $\gamma$-Hölder continuous path $x$. Furthermore, if $b$ is a measurable function, and $z$ is path of finite $p$-variation, then set $\Gamma_{s, t}\left(y_{s}\right)=\int_{s}^{t} b\left(y_{s}+z_{r}\right) \mathrm{d} r$. In this case, it is readily checked that the integral coincides with the classical Riemann integral

$$
\int_{0}^{t} b\left(y_{r}+z_{r}\right) \mathrm{d} r=\int_{0}^{t} \Gamma_{\mathrm{d} r}\left(y_{r}\right) .
$$

See [12] for a comprehensive introduction and discussion of the nonlinear integral.

For self-containedness, we include a proof of Lemma 33. The existence and uniqueness of these equations has been proven in $[6,15]$, and we refer to these references for a full account on these results.

Proof of Lemma 33 This proof follows along the lines of [15, Lemma 30], and thus, we only give here a shorter recollection of the most important details. Let $\beta \in\left(\frac{1}{\kappa}, \gamma\right)$ where we recall that $\kappa>\frac{1}{\gamma}$ by assumption, and let $\mathfrak{S}_{T}: \mathcal{C}_{T}^{\beta}\left(\mathbb{R}^{d}\right) \rightarrow \mathcal{C}_{T}^{\beta}\left(\mathbb{R}^{d}\right)$ be the solution map given by

$$
\mathfrak{S}_{T}(y):=\left\{\xi+\int_{0}^{t} \Gamma_{\mathrm{d} r}\left(y_{r}\right) \mid t \in[0, T]\right\} .
$$

Let $\mathscr{B}_{T}(\xi) \subset \mathcal{C}_{T}^{\beta}\left(\mathbb{R}^{d}\right)$ be a unit ball centered at $\xi \in \mathbb{R}^{d}$. In order to prove existence and uniqueness of (5.2), we will begin to show that there exists a $\tau>0$ such that $\mathfrak{S}_{\tau}$ leaves the unit ball $\mathscr{B}_{\tau}(\xi)$ invariant. In the second step, we will show that there exists a $\tau^{\prime}>0$ such that the solution map $\mathfrak{S}_{\tau^{\prime}}$ is a contraction on the unit ball $\mathscr{B}_{\tau^{\prime}}(\xi)$. It then follows by Picard's fixed point theorem that a unique solution exists in the unit ball $\mathscr{B}_{\bar{\tau}}(\xi)$ for $\bar{\tau}=\tau \wedge \tau^{\prime}$. In the end, since $\xi \mapsto \Gamma(\xi)$ is globally bounded, we can iterate the solution to the intervals $[k \bar{\tau},(k+1) \bar{\tau} \wedge T]$ for $k \in \mathbb{N}$.

We begin to show the invariance. By application of (B.3) and (i) in (5.1), it follows that for $y \in \mathscr{B}_{\tau}(\xi)$

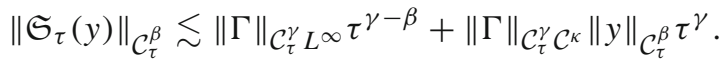

Using that $y \in \mathscr{B}_{\tau}(\xi)$, and thus in particular $\|y\|_{\mathcal{C}_{\tau}^{\beta}} \leq 1$, it follows that

$$
\left\|\mathfrak{S}_{\tau}(y)\right\|_{\mathcal{C}_{\tau}^{\beta}} \lesssim\|\Gamma\|_{\mathcal{C}_{\tau}^{\gamma} \mathcal{C}^{\kappa}} \tau^{\gamma-\beta}
$$


By choosing $\tau>0$ sufficiently small, we obtain $\left\|\mathfrak{S}_{\tau}(y)\right\|_{\mathcal{C}_{\tau}^{\beta}} \leq 1$, and thus, $\mathfrak{S}_{\tau}$ leaves the unit ball $\mathscr{B}_{\tau}(\xi)$ invariant. We continue to prove the contraction property. Applying (B.3), it follows from Lemma 38 that for $y, z \in \mathscr{B}_{\tau^{\prime}}(\xi)$ we have

$$
\begin{aligned}
\left\|\mathfrak{S}_{\tau^{\prime}}(y)-\mathfrak{S}_{\tau^{\prime}}(z)\right\|_{\mathcal{C}_{\tau^{\prime}}^{\beta}} & \lesssim\|\Gamma(y)-\Gamma(z)\|_{\mathcal{C}_{\tau^{\prime}}^{\beta}}+\left\|\int_{0}\left(\Gamma_{\mathrm{d} r}(y)-\Gamma_{\mathrm{d} r}(z)\right)-(\Gamma(y)-\Gamma(z))\right\|_{\mathcal{C}_{\tau^{\prime}}^{\beta}} \\
& \lesssim\|\Gamma(y)-\Gamma(z)\|_{\mathcal{C}_{\tau^{\prime}}^{\beta}}+\|\delta[\Gamma(y)-\Gamma(z)]\|_{\mathcal{C}^{\beta^{\prime}}}\left(\tau^{\prime}\right)^{\beta^{\prime}-\beta}
\end{aligned}
$$

for some $\beta^{\prime}>1$. We may assume that $z_{0}=y_{0}=\xi$. For the first term on the right hand side, it follows by assumption (B.1) (ii) that

$$
\|\Gamma(y)-\Gamma(z)\|_{\mathcal{C}_{\tau^{\prime}}^{\beta}} \lesssim \Gamma\left(\tau^{\prime}\right)^{\gamma-\beta}\|y-z\|_{\mathcal{C}_{\tau^{\prime}}^{\beta}},
$$

where we have used that $y_{0}-z_{0}=0$. For the second term on the right hand side of (B.6), we appeal to the proof of the nonlinear Young integral in Lemma 38, and we will need to show that the action of the $\delta$-operator on the integrand $\Xi_{s, t}:=\Gamma_{s, t}\left(y_{s}\right)-\Gamma_{s, t}\left(z_{s}\right)$ is sufficiently regular and has a contractive property. That is, we will prove that for $(s, u, t) \in \Delta_{\tau^{\prime}}^{3}$, the following inequality holds $\left|\delta_{u} \Xi_{s, t}\right| \lesssim|t-s|^{\mu}\|y-z\|_{\mathcal{C}^{\beta}}$. By the fundamental theorem of calculus, it follows that

$$
\Xi_{s, t}=\int_{0}^{1} \nabla \Gamma_{s, t}\left(\rho y_{s}+(1-\rho) z_{s}\right) d \rho\left(y_{s}-z_{s}\right) .
$$

By the same algebraic manipulations as used in (B.5), it is readily checked that for $(s, u, t) \in \Delta_{T}^{3}$ we have

$$
\begin{aligned}
\delta_{u} \Xi_{s, t}= & \int_{0}^{1} \nabla \Gamma_{u, t}\left(\rho y_{s}+(1-\rho) z_{s}\right)\left(y_{s}-z_{s}\right) \\
& \quad-\nabla \Gamma_{u, t}\left(\rho y_{u}+(1-\rho) z_{u}\right) d \rho\left(y_{u}-z_{u}\right) d \rho .
\end{aligned}
$$

By addition and subtraction of $\nabla \Gamma_{u, t}\left(\rho y_{s}+(1-\rho) z_{s}\right)\left(y_{u}-z_{u}\right)$ inside the above integral, invoking (i) of (5.1) and using that $\kappa \geq 1$, we begin to observe that

$$
\left|\nabla \Gamma_{u, t}\left(\rho y_{s}+(1-\rho) z_{s}\right)\left[\left(y_{s}-z_{s}\right)-\left(y_{u}-z_{u}\right)\right]\right| \lesssim\|\Gamma\|_{\mathcal{C}_{T}^{\gamma} \mathcal{C}^{\kappa}}\|y-z\|_{\mathcal{C}_{\tau^{\prime}}^{\beta}}|t-s|^{\gamma+\beta}
$$

Furthermore, invoking (iii) of (5.1), it follows that

$$
\begin{aligned}
& \left|\left[\nabla \Gamma_{u, t}\left(\rho y_{s}+(1-\rho) z_{s}\right)-\nabla \Gamma_{u, t}\left(\rho y_{u}+(1-\rho) z_{u}\right)\right]\left(y_{u}-z_{u}\right)\right| \\
& \lesssim\left[\|y\|_{\mathcal{C}_{\tau^{\prime}}^{\beta}} \vee\|z\|_{\mathcal{C}_{\tau^{\prime}}^{\beta}}\right]^{\kappa-1}\|\Gamma\|_{\mathcal{C}_{T}^{\gamma} \mathcal{C}^{\kappa}}|t-u|^{\gamma}|u-s|^{\beta(\kappa-1)}\left(\left|y_{0}-z_{0}\right|+\|y-z\|_{\mathcal{C}_{\tau^{\prime}}^{\beta}}\right) .
\end{aligned}
$$


Due to the assumption that $\beta \in\left(\frac{1}{\kappa}, \gamma\right)$ it follows that $\beta(\kappa-1)+\gamma>1$. Combining (B.8) and (B.9), it follows that for $y, z \in \mathscr{B}_{\tau^{\prime}}(\xi)$ with $z_{0}=y_{0}=\xi$ we set $\beta^{\prime}=$ $\beta(\kappa-1)+\gamma$ and we have

$$
\left\|\delta_{u} \Xi_{s, t}\right\|_{\mathcal{C}_{\tau^{\prime}}^{\beta^{\prime}}} \lesssim \Gamma\|y-z\|_{\mathcal{C}_{\tau^{\prime}}^{\beta}}
$$

Thus, inserting (B.7) and (B.10) into the right hand side of (B.6), we obtain the inequality

$$
\left\|\mathfrak{S}_{\tau^{\prime}}(y)-\mathfrak{S}_{\tau^{\prime}}(z)\right\|_{\mathcal{C}_{\tau^{\prime}}^{\beta}} \lesssim \Gamma\|y-z\|_{\mathcal{C}_{\tau^{\prime}}^{\beta}}\left(\tau^{\prime}\right)^{\gamma-\beta}
$$

By choosing $\tau^{\prime}>0$ small enough, it is clear that the solution map $\mathfrak{S}_{\tau^{\prime}}$ is a contraction on the ball $\mathscr{B}_{\tau^{\prime}}(\xi)$. Note in particular that the contraction bound is independent on the initial data, due to the assumption of boundedness of the derivatives of $\Gamma$ (recall that $\mathcal{C}_{\tau}^{\gamma} \simeq C_{b}^{\gamma}([0, \tau])$ when $\left.\gamma \in(0,1)\right)$.

It follows that $\mathfrak{S}_{\tau \wedge \tau^{\prime}}$ is a contraction and leaves the ball $\mathscr{B}_{\tau^{\prime}}(\xi)$ invariant, and it follows by Picard's fixed point theorem that there exists a unique solution to (5.2) on in $\mathscr{B}_{\tau^{\prime}}(\xi)$. By standard procedures, one can now iterate the solution to the whole interval $[0, T]$, and we ask the patient reader to consult [11, Section 8.3] for further details on this part. At last we note that the solution is indeed contained in the space $\mathcal{C}_{T}^{\gamma}$. Indeed, assume $y \in \mathcal{C}_{T}^{\beta}$ satisfies (5.2). Using the inequality in (B.4), the following inequality holds

$$
\left|y_{s, t}\right|=\left|\int_{s}^{t} \Gamma_{\mathrm{d} r}\left(y_{r}\right)\right| \lesssim\left|\Gamma_{s, t}\left(y_{s}\right)\right|+\|\Gamma\|_{\mathcal{C}_{T}^{\gamma} \mathcal{C}^{\kappa}}\|y\|_{\mathcal{C}_{T}^{\beta}}|t-s|^{\gamma+\beta} \lesssim y, \Gamma, T|t-s|^{\gamma},
$$

and it follows that $y \in \mathcal{C}_{T}^{\gamma}$. This concludes our proof.

\section{References}

1. Applebaum, D.: Lévy Processes and Stochastic Calculus, volume 93 of Cambridge studies in advanced mathematics. Cambridge University Press, Cambridge (2004)

2. Athreya, S., Butkovsky, O., Oleg, M.: Strong existence and uniqueness for stable stochastic differential equations with distributional drift. Ann. Probab. 48(1), 178-210 (2020)

3. Bahouri, H., Chemin, J.-Y., Danchin, R.: Fourier analysis and nonlinear partial differential equations. Springer, Berlin (2011)

4. Bass, R.F.: Chen, Zhenqing: Stochastic differential equations for Dirichlet processes. Probab. Theory Related Fields 121(3), 422-446 (2001)

5. Berman, S.M.: Local nondeterminism and local times of Gaussian processes. Bull. Am. Math. Soc. 79, 475-477 (1973)

6. Catellier, R., Gubinelli, M.: Averaging along irregular curves and regularisation of ODEs. Stochastic Process. Appl. 126(8), 2323-2366 (2016)

7. Davie, A. M.: Uniqueness of Solutions of Stochastic Differential Equations. International Mathematics Research Notices (2007), 01 2007. rnm124 
8. de Raynal, P.E.C., Menozzi, S., Priola, E.: Weak well-posedness of multidimensional stable driven sdes in the critical case. Stochastics Dyn. 20(06), 204 (2020)

9. Flandoli, F.: Regularizing properties of brownian paths and a result of davie. Stochatics Dyn. 2-3(11), 323-331 (2011)

10. Flandoli, F.: Issoglio, Elena, Russo, Francesco: Multidimensional stochastic differential equations with distributional drift. Trans. Am. Math. Soc. 369(3), 1665-1688 (2017)

11. Peter, K.F., Martin H.: A course on rough paths. Universitext. Springer, Cham (2014). With an introduction to regularity structures

12. Galeati, L.: Gubinelli. Noiseless regularisation by noise, Massimiliano (2020)

13. Galeati, L.G.: Prevalence of rho-irregularity and related properties, Massimiliano (2020)

14. Geman, D., Horowitz, J.: Occupation times for smooth stationary processes. Ann. Probab. 1(1), 131137 (1973)

15. Harang, F.A.: Perkowski. C-infinity regularization of odes perturbed by noise, Nicolas (2020)

16. Yaozhong, H., Lê, K.: Nonlinear Young integrals and differential systems in hölder media. Trans. Am. Math. Soc. 369(3), 1935-2002 (2017)

17. Kang, J., Kim, P.: Tangential limits for harmonic functions with respect to $\phi(\delta)$ : stable and beyond. Potential Anal. 42, 629-644 (2015)

18. Krylov, N.V.: Röckner, Michael: Strong solutions of stochastic equations with singular time dependent drift. Probab. Theory Related Fields 131(2), 154-196 (2005)

19. Lê, K.: A stochastic sewing lemma and applications. Electron. J. Probab. 25, 420 (2020)

20. Nolan, J.P.: Local nondeterminism and local times for stable processes. Probab. Theory Related Fields 82(3), 387-410 (1989)

21. Pitt, L.D.: Local times for Gaussian vector fields. Indiana Univ. Math. J. 27(2), 309-330 (1978)

22. Priola, E.: Pathwise uniqueness for singular sdes driven by stable processes. Osaka J. Math. 49(2), 421-447 (2012)

23. Priola, E.: Davie type uniqueness for a class of sdes with jumps. Ann. Inst. H. Poincaré Probab. Statist. 54(2), 694-725 (2018)

24. Protter, P.E.: Stochastic integration and differential equations, 2nd edn. Springer, Berlin (2004)

25. Rajput, B.S.: Spectral representations of infinitely divisible processes. Probab. Theory Related Fields 82, 451-487 (1989)

26. Rosinski, J.: On stochastic integral representation of stable processes with sample paths in banach spaces. J. Multivariate Anal. 20(2), 277-302 (1986)

27. Samorodnitsky, G., Taggu, M.S.: Stable non-Gaussian random processes: stochastic models with infinite variance. Chapman Hall, New York (1994)

28. Sato, K.: Lévy Processes and Intensity Divisible Distributions, volume 68 of Cambridge studies in advanced mathematics. Cambridge University Press, Cambridge (1990)

29. Xiao, Y.: Properties of local-nondeterminism of Gaussian and stable random fields and their applications. Ann. Fac. Sci. Toulouse Math. (6) 15(1), 157-193 (2006)

30. Zhang, X., Zhao, G.: Heat kernel and ergodicity of sdes with distributional drifts. arXiv:1710.10537 (2017)

Publisher's Note Springer Nature remains neutral with regard to jurisdictional claims in published maps and institutional affiliations. 